\title{
Rap1 is involved in the signal transduction of myelin-associated glycoprotein
}

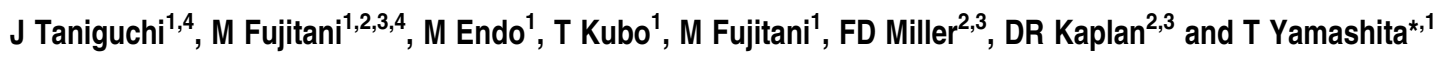

Myelin-associated glycoprotein (MAG) is a well-characterized axon growth inhibitor in the adult vertebrate nervous system. Several signals that play roles in inhibiting axon growth have been identified. Here, we report that soluble MAG induces activation of Rap1 in postnatal cerebellar granule neurons (CGNs) and dorsal root ganglion (DRG) neurons. The p75 receptor associates with activated Rap1 and is internalized in response to MAG. After MAG is applied to the distal axons of the sciatic nerves, the activated Rap1, internalized p75 receptor, and MAG are retrogradely trafficked via axons to the cell bodies of the DRG neurons. Rap1 activity is required for survival of the DRG neurons as well as CGNs when treated with MAG. The transport of the signaling complex containing the p75 receptor and Rap1 may play a role in the effect of MAG.

Cell Death and Differentiation (2008) 15, 408-419; doi:10.1038/sj.cdd.4402278; published online 30 November 2007

\begin{abstract}
Myelin-associated glycoprotein (MAG) is a member of the I-type lectin subgroup of the immunoglobulin (Ig) gene superfamily and is expressed exclusively by myelinating cells where it is enriched in the adaxonal membrane of the myelin internode. MAG inhibits neurite outgrowth from the adult dorsal root ganglion (DRG) and the postnatal ages of the cerebellar, retinal, spinal, hippocampal, and superior cervical ganglion neurons. ${ }^{1,2}$ To date, two additional neurite growth inhibitors that are expressed by oligodendrocytes and myelinated fiber tracts have been identified. ${ }^{3}$ These are Nogo and oligodendrocyte-myelin glycoprotein. All these proteins act on neurons through the Nogo receptor complex. This is a trimolecular complex comprising the Nogo receptor, Lingo-1, and p75/Troy. ${ }^{4} \mathrm{~A}$ key intracellular effector for neurite growth inhibitory signaling by myelin was previously shown to be the small guanine nucleoside triphosphatase, RhoA. In its active GTP-bound form, RhoA rigidifies the actin cytoskeleton, thereby inhibiting axon elongation and mediating growth cone collapse. RhoA is activated by these proteins through a p75dependent mechanism, thus inhibiting neurite outgrowth from the postnatal sensory neurons and cerebellar neurons. ${ }^{5,6}$ It was also demonstrated that conventional protein kinase $\mathrm{C}$ (PKC), including PKC $-\alpha,-\beta$, and $-\gamma$, was activated by the neurite growth inhibitory proteins, and the inhibition of conventional PKC eliminated the effect of these proteins on neurite outgrowth. ${ }^{7,8}$ In addition, phosphorylation of the
\end{abstract}

epidermal growth factor (EGF) receptor is triggered by these myelin-derived inhibitors and is necessary for the inhibitory effect. ${ }^{9}$ These multiple signals are responsible for the effects of the myelin-derived inhibitors at least in vitro.

In addition to the role of MAG in axon regeneration, experiments with MAG-deficient mice implicate this protein in regulating axonal caliber and the levels of neurofilament spacing in mature myelinated fibers. ${ }^{10}$ MAG is suggested to modulate the maturation and viability of myelinated axons. These findings suggest the diverse functions of MAG in the nervous system.

In the course of identifying signals downstream of MAG, we found that Rap1 is activated in response to soluble MAG. Rap1 is a member of the Ras family of small guanine nucleotidebinding proteins ( $G$ proteins), which activate and inhibit several proteins in a GTP-dependent manner. ${ }^{11}$ Rap1 is implicated in a particularly wide range of biological processes, from cell proliferation and differentiation to cell adhesion. Rap1 opposes the actions of Ras, including the regulation of cell growth and differentiation, integrin-dependent responses, and synaptic plasticity. Furthermore, recent evidence has demonstrated that Rap1, like Ras, can activate the mitogen-activated protein kinase cascade in several cell types. In this study, we demonstrate that MAG activates Rap1 through a p75dependent mechanism and that Rap1 activity is required for the cell survival of neurons following stimulation with MAG.

\footnotetext{
${ }^{1}$ Department of Neurobiology, Graduate School of Medicine, Chiba University, Chuo-ku, Chiba, Japan; ${ }^{2}$ Cell Biology, Developmental and Stem Cell Biology Program, The Hospital for Sick Children, Toronto, Ontario, Canada and ${ }^{3}$ Department of Medical Genetics and Microbiology, University of Toronto, Toronto, Ontario, Canada ${ }^{*}$ Corresponding author: T Yamashita, Department of Neurobiology, Graduate School of Medicine, Chiba University, 1-8-1 Inohana, Chuo-ku, Chiba 260-8670, Japan. Tel: + 8143 2262024; Fax: + 8143 2262025; E-mail: t-yamashita@faculty.chiba-u.jp

${ }^{4}$ These authors contributed equally to the work.

Keywords: myelin; neuron; axon; Rap; p75; GTPase

Abbreviations: BSA, bovine serum albumin; CGN, cerebellar granule neurons; DA, dominant active; DMEM, Dulbecco's modified Eagle's medium; DN, dominant negative; DRG, dorsal root ganglion; EGF, epidermal growth factor; ERK, extracellular signal-regulated kinases; GST, the glutathione S-transferase; HA, hemagglutinin; HD, helical domain; ICD, intracellular domain; Ig, immunoglobulin; MAG, myelin-associated glycoprotein; MAG-Fc, the extracellular domain of MAG fused to the FC region of human IgG; NGF, nerve growth factor; PB, phosphate buffer; PFA, paraformaldehyde; PKC, protein kinase C; RalGDS, Ral guanine nucleotide dissociation stimulator; SDS, sodium dodecyl sulfate; SFM, serum-free media; TUNEL, terminal deoxynucleotidyl-transferase-mediated dUTP nick end labeling

Received 23.4.07; revised 24.9.07; accepted 26.10.07; Edited by CS Duckett; published online 30.11.07
} 


\section{Results}

MAG induces activation of Rap1 in postnatal cerebellar granule neurons. To examine whether MAG influences the activity of Rap1 in myelin-responsive neurons, we treated serum-starved cerebellar granule neurons (CGNs) from postnatal day 7 (P7) rats with recombinant soluble MAG and assessed the activity of endogenous Rap1 by using a pull-down assay. The effector protein Ral guanine nucleotide dissociation stimulator (RalGDS) was used to specifically precipitate GTP-bound form of Rap1. A soluble chimeric form of MAG, consisting of the extracellular domain of MAG fused to the Fc region of human IgG (MAG-Fc), was used. It was previously shown that soluble MAG, released in abundance from myelin and found in vivo, and MAG-Fc could potently inhibit axonal growth. ${ }^{12,13}$ Our results revealed that soluble MAG-Fc $(25 \mu \mathrm{g} / \mathrm{ml})$ triggered Rap1 activation from $10 \mathrm{~min}$ to $4 \mathrm{~h}$ after its addition into the medium (Figure 1a). In contrast, the activity of Ras, another member of the Ras family of small $G$ proteins, was suppressed transiently and returned to the control level at $4 \mathrm{~h}$ (Figure 1a). These results support the notion that Rap1 opposes the actions of Ras. We then employed CGNs (P7) from mice carrying a mutation in the p75 gene to determine whether p75 is required for Rap1 activation by MAG-Fc. Although Rap1 was activated in the CGNs from wild-type mice $30 \mathrm{~min}$ after the MAG-Fc treatment, Rap1 activation by MAG-Fc could not be observed in CGNs from mice carrying a mutation in the p75 gene (Figure 1b). Thus, activation of Rap 1 by MAG-Fc is dependent on $\mathrm{p} 75$.

Rap1 associates with p75 intracellular domain. We next examined whether Rap1 associates with p75. Hemagglutinin (HA)-tagged full-length $p 75$ and various forms of myc-tagged Rap1A or $-B$ were transfected into 293 T cells. Rap1A is ubiquitously expressed in tissues, whereas Rap1B is predominantly expressed in platelets. ${ }^{11}$ In the p75 precipitates, an anti-myc antibody revealed the presence of the dominant-active (DA) form as well as of the wild-type but not of the dominant-negative (DN) form of Rap1A (Figure 1c). Any form of Rap1B was not co-immunoprecipitated with p75 (Figure 1c). These results suggest that the GTP-bound form of Rap1A predominantly associates with p75 in the transfected 293T cells. However, we could not conclude that the DN form of Rap1A did not interact with p75. The expression level of the DN form was low, although we tried to express it at the equivalent level. We then examined the interaction of endogenous p75 with Rap1 by using lysates prepared from rat CGNs (P7). An association of endogenous p75 with Rap1 was observed when the cells were treated with MAG-Fc but not in the absence of MAG-Fc (Figure 1d). This result is consistent with the observation that Rap1 was activated in response to MAG-Fc (Figure 1a) and that the active form of Rap1 predominantly interacted with p75 (Figure 1c). These findings demonstrate that Rap1 is activated in CGNs when treated with MAG-Fc and that Rap1 associates with p75 presumably in the GTP-bound form.

We next examined the direct physical interaction of p75 with Rap1 using purified recombinant proteins. Bacterially produced His-tagged Rap1A in the GDP-bound, GTP-bound, or in the nucleotide-depleted state was incubated with the glutathione $S$-transferase (GST) fusion protein containing the intracellular domain (ICD) of p75 (GST-p75ICD). We observed the interaction of GST-p75ICD with Rap1A predominantly in the GTP-bound or nucleotide-free state but only weakly in the GDP-bound state (Figure 1e). This pattern of association is similar to that of Rap1A and its effector RalGDS (Figure 1e). We then assessed the structural basis of the interaction between Rap1A and P75ICD. Experiments with partial fragments of p75ICD showed that constructs that included the 'linker region' but not those that included the six $\alpha$ helices of p75 interacted with Rap1A (Figure 1f). However, the interaction of Rap1A with $\mathrm{p} 75 \mathrm{ICD}$ was stronger than that with the linker region (LR), suggesting that the helical domain (HD) might enhance the interaction. We previously showed that RhoGDI in complex with RhoA associates with the HD of p75. ${ }^{14}$ Therefore, our mapping study shows distinct binding sites for Rap1 and RhoA-RhoGDI.

Internalization and trafficking of p75. We next hypothesized that p75 is internalized and trafficked after stimulation with MAG-Fc in the neurons. Although the internalization of p75 by neurotrophin binding has been demonstrated, ${ }^{15,16}$ there is no evidence for MAG-induced p75 internalization. We addressed this question by using DRG neurons as CGNs are too small for visualization and the DRG neurons constitute a well-established experimental system to study the effects of MAG. p75 was labeled with the MC192 monoclonal antibody (MC192). ${ }^{17}$ MC192 binds to an extracellular epitope on rat p75 that is distinct from the binding site for nerve growth factor (NGF). The DRG neurons were incubated for $30 \mathrm{~min}$ with Alexa 594-labeled MC192 followed by incubation with Alexa 488-labeled MAG-Fc at a concentration of $25 \mu \mathrm{g} / \mathrm{ml}$ in serum-free media (SFM) at $4^{\circ} \mathrm{C}$. After washing, the neurons were then incubated for $60 \mathrm{~min}$ at $37^{\circ} \mathrm{C}$ in SFM followed by two washes in ice-cold acid to eliminate surface binding of MAG-Fc. Figure 2a shows that MAG-Fc- (green) and MC192-labeled p75 (red) were internalized and colocalized in the cell bodies and the neurites of the DRG neurons (also see Supplementary Figure 1S). Although majority of the signals were colocalized, some of the MAG-Fc signals were negative for the MC192labeled $p 75$, raising the possibility that another receptor for MAG, Troy, may play a role.

We then used CGNs to confirm these results by biochemical analysis. The CGNs (P7) were treated with MAG-Fc and MC192 for $30 \mathrm{~min}$ and were then incubated in MAG-Fcfree media for up to $60 \mathrm{~min}$. The result indicated that MAG-FC was present in the cytosolic fraction of the CGNs at 15-60 min after the elimination of MAG-Fc from the medium (Figure 2b). MC192 was co-internalized in a similar time course.

We next performed time-lapse video fluorescence microscopy to observe the movement of the labeled p75 in the DRG neurons. The DRG neurons from P7 rats were labeled with Alexa 594-conjugated MC192 for $30 \mathrm{~min}$, washed, and then treated with MAG-Fc $(25 \mu \mathrm{g} / \mathrm{ml})$. Figure 2c shows the fluorescence images of Alexa 594-conjugated MC192 at 20-min intervals. Diffuse fluorescence at the surface of the neurons was observed in the growth cones (Figure 2c, d) or 
a

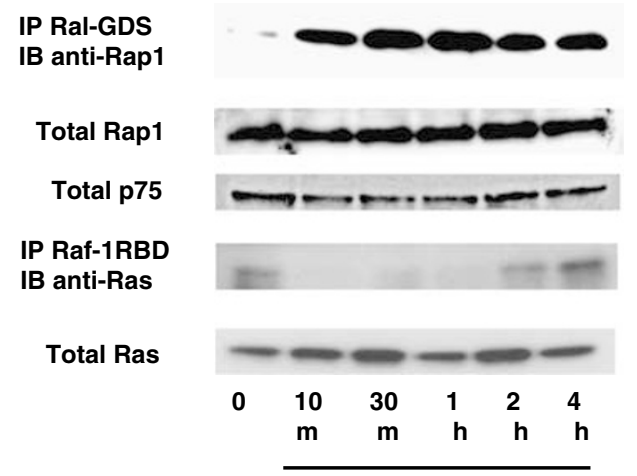

MAG-Fc

b IP Ral-GDS

IB anti-Rap1

Total Rap1

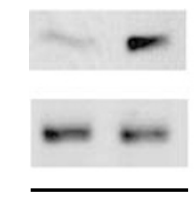

p75+/t

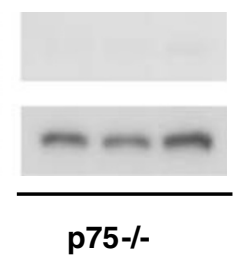

MAG-Fc

$0 \quad 30$

0

30

$60 \mathrm{~min}$
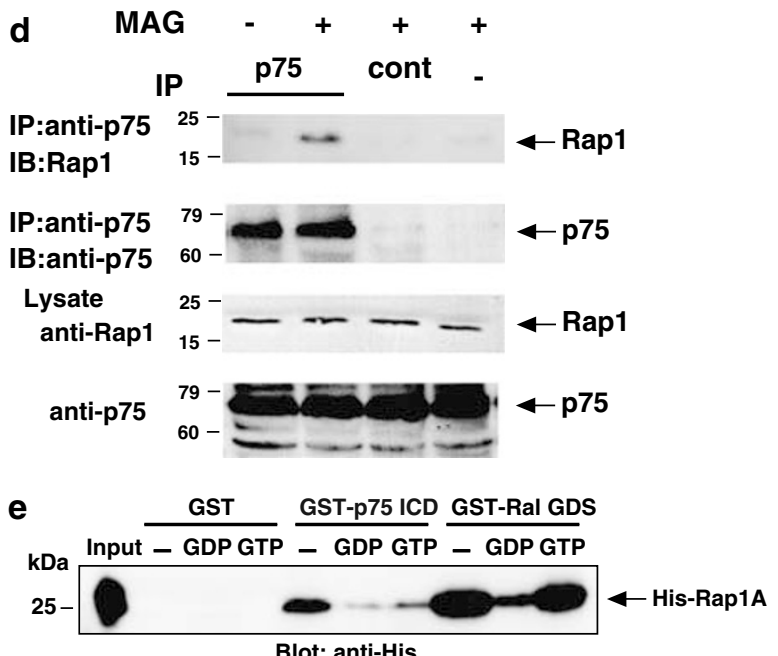

Blot: anti-His

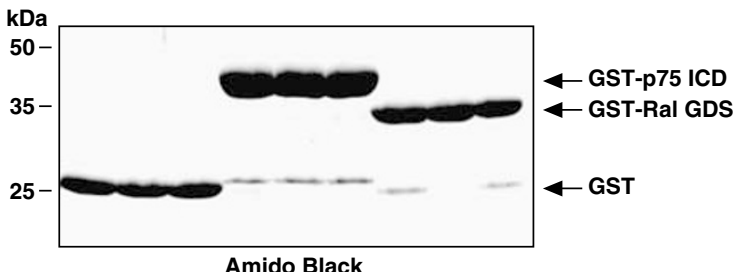

C
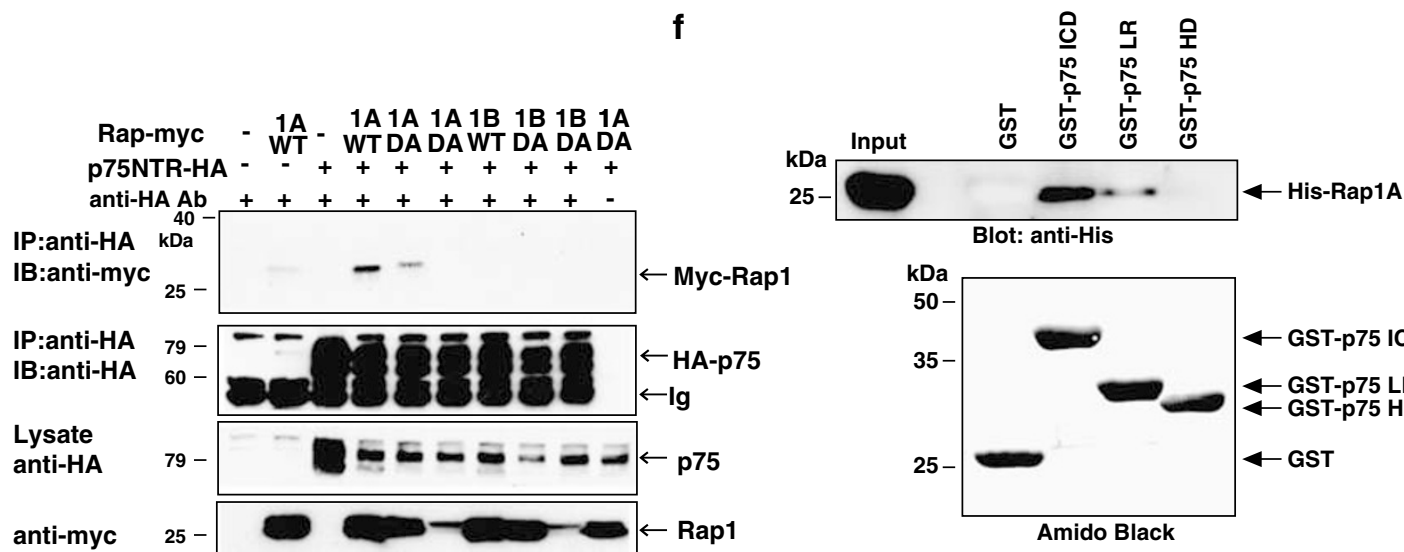

IB:anti-myc $25-\quad \leftarrow$ Myc-Rap1

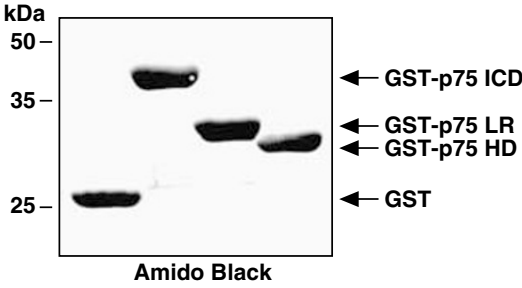

Figure 1 Involvement of Rap1 in MAG-p75 signaling. (a) Affinity precipitation of Rap1 and Ras in postnatal CGNs. Rap1 activity increased from $10 \mathrm{~min}$ to $4 \mathrm{~h}$ after the addition of MAG-Fc $(25 \mu \mathrm{g} / \mathrm{ml})$. The second and third panels show the total amount of Rap1 and p75, respectively, in the lysates. The fourth panel shows the amount of Ras precipitated by the RBD of Raf1 (Raf1RBD), and the bottom panel shows the total amount of Ras in the lysates. Ras activity was transiently suppressed by MAG-Fc. The result was reproducible $(n=3)$. (b) Rap1 was not activated in the CGNs from mice carrying a mutation in the p75 gene. The CGNs from wild-type mice $\left(p 75^{+/+}\right)$or mice carrying a mutation in the $p 75$ gene $\left(\mathrm{p} 75^{-1-}\right)$ were treated with MAG-Fc $(25 \mu \mathrm{g} / \mathrm{ml})$ for the indicated times, and the Rap1 activity was examined. The result was reproducible $(n=4)$. (c) Association of p75 with Rap1A in the transfected 293T cells. Immunoprecipitation of the HA-tagged p75 (p75NTR-HA) followed by blotting with the anti-myc antibody. The dominant-active form and the wild type of Rap1A but not of Rap1B were co-immunoprecipitated with p75NTR-HA. The dominant-negative form of Rap1A did not interact with p75. 1A, Rap1A; 1B, Rap1B; WT, wild-type; DA, dominant-active form; DN, dominant-negative form; IP, immunoprecipitation; IB, immunoblot. (d) Association of endogenous p75 with Rap 1 in the CGNs. The CGNs were treated with or without MAG-Fc for 30 min. p75 was immunoprecipitated with the anti-p75 antibody from the lysates prepared from the CGNs and was blotted with the anti-Rap1 antibody. The association was found in the CGNs that were treated with MAG-Fc. IP-p75, immunoprecipitation with MC192; IPcont, immunoprecipitation with mouse monoclonal IgG. (e) p75 directly associates with Rap1. Co-precipitation of GST-p75ICD with recombinant His-Rap1A. Interaction was examined by Western blot analysis of the precipitates produced with the purified GST-p75ICD and glutathione-Sepharose beads. The anti-His antibody revealed the presence of a His-Rap1A predominantly in the nucleotide-free and GTP-bound states (top). GST-RalGDS was used as a positive control. The bottom panel shows amido black staining The negative image represents high levels of the GST fusion proteins. (f) p75LR is required for the interaction of p75 with Rap1A. Co-precipitation of recombinant His-Rap1A with GST-p75ICD, GST-p75LR, or GST-p75HD. GST-fused proteins were precipitated, electrophoresed, and blotted with anti-His antibody (top). The bottom panel shows amido black staining. The negative image represents high levels of the GST fusion proteins. LR, linker region; HD, helical domain

neurites (Figure 2f) of the DRG neurons when the assay was begun. However, we observed some fluorescence particles in the growth cones at 20 min (Figure 2e). A number of fluorescence signals with punctate patterns in the neurites and the cell bodies were observed beyond $40 \mathrm{~min}$ (Figure 2c, g). Although these puncta in the neurites moved in both directions, more puncta moved retrogradely than anterogradely in the neurites (Supplementary movie). The quantification 
a

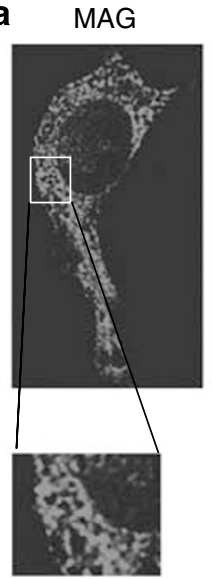

p75

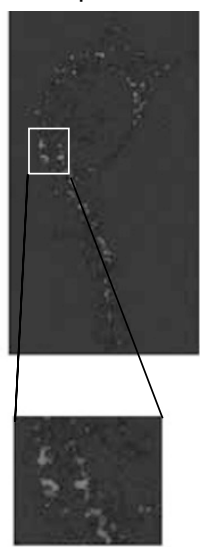

merge

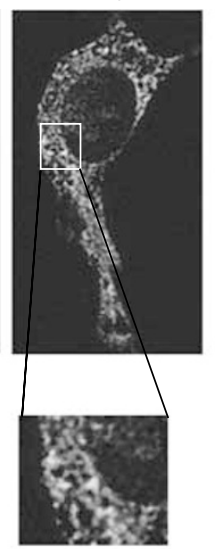

b

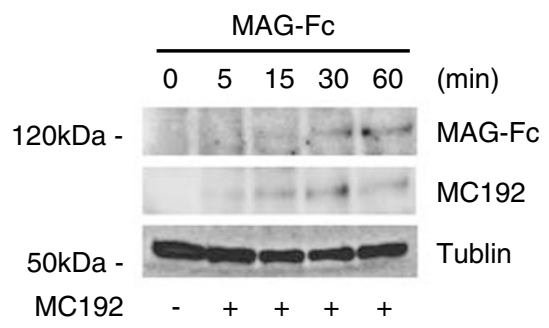

c

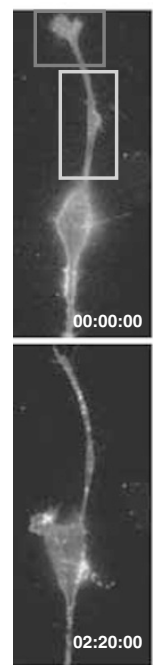

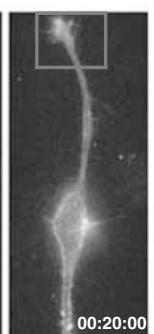

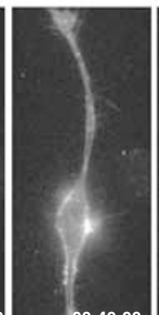

00:40:00
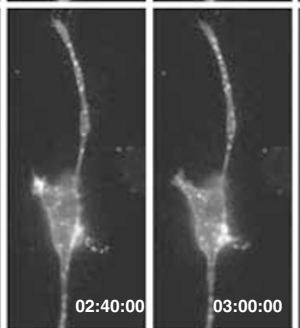
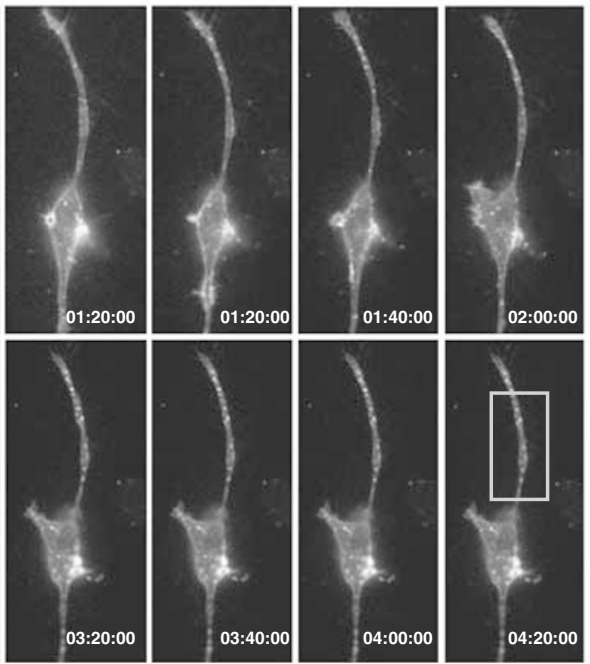

d

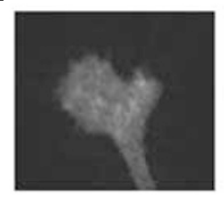

f

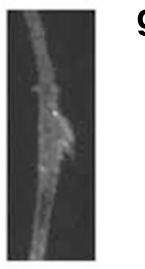

e
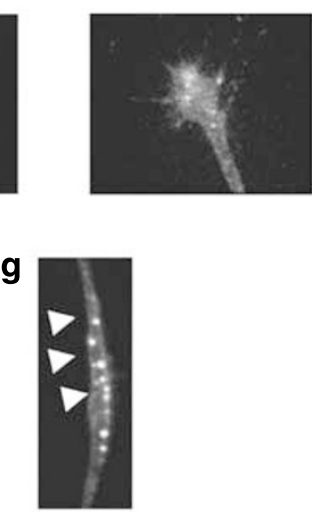

h

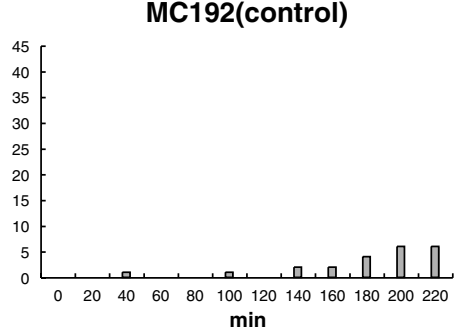

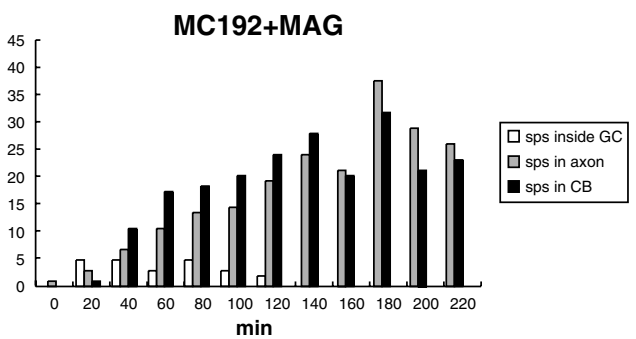

Figure 2 Internalization and trafficking of p75 and MAG-Fc into the neurons. (a) Colocalization of the signals for the internalized MAG-Fc and p75. The DRG neurons were incubated for $30 \mathrm{~min}$ with Alexa 594-labeled MC192 followed by incubation with Alexa 488-labeled or unlabelled MAG-Fc in SFM at $4^{\circ} \mathrm{C}$. After washing, the neurons were incubated for $60 \mathrm{~min}$ in SFM followed by two washes in ice-cold acid to eliminate surface binding of MAG-Fc and fixation in 4\% PFA. Green, MAG-Fc; red, MC192; Merge, merged figures. (b) The CGNs were treated with MAG-Fc and MC192 at $4{ }^{\circ} \mathrm{C}$ for $30 \mathrm{~min}$ and then incubated in prewarmed SFM without MAG-Fc for the indicated times. MAGFc or MC192 attached to the membrane surface was removed by acid wash. Western blot shows the amount of internalized MAG-Fc (top panel) and MC192 (middle) in the CGNs. The bottom panel demonstrates the amount of tubulin in the lysates. (c) MAG-Fc induces retrograde or anterograde movement of p75 in the DRG neurons. P7 DRG neurons were labeled with Alexa 594-labeled MC192 followed by treatment with MAG-Fc $(25 \mu \mathrm{g} / \mathrm{ml})$. The labeled p75 was recorded by time-lapse video fluorescence microscopy. Each frame shows the fluorescence image of Alexa 594 at 20 -min intervals. (d-g) High-magnification images at time 0 in the growth cone (d) and the neurite (f), at $20 \mathrm{~min}$ in the growth cone $(\mathbf{e})$, and at $4 \mathrm{~h}$ and $20 \mathrm{~min}$ in the neurite $(\mathrm{g})$. The square area at $4 \mathrm{~h}$ and $20 \mathrm{~min}$ in $(\mathbf{c})$ corresponds to $(\mathbf{g})$. Note multiple fluorescence particles in the growth cone as well as the neurites after the addition of MAG-Fc. (h) Quantification of the number of fluorescence particles for the labeled p75. The number of fluorescence particles in a growth cone (GC), a neurite, (axon) or a cell body (CB) was measured at each time point, and the average number $(n=50)$ is shown. A similar result was obtained in every neuron examined. MC192 + MAG, with MAG-Fc treatment; MC192, without MAG-Fc treatment; Sps, small particles

of the data demonstrated that the punctate signals in the cell bodies and neurites increased gradually up to $4 \mathrm{~h}$ and $20 \mathrm{~min}$ (Figure 2h). We observed only diffuse fluorescence and no movement of the signals in the DRG neurons that were not treated with MAG-Fc (Figure 2h; Supplementary Figure 2S). These findings suggest that the internalized p75 was trafficked to the cell bodies via neurites after the cells were treated with MAG-Fc. 
p75 is retrogradely trafficked to the cell bodies of the sciatic nerves. The above results suggest that p75 transmits a retrograde signal. If Rap1 is activated by MAGFc at the distal axons, this activated Rap1 may be trafficked to the cell bodies. To assess this, we developed a method in which the isolated DRG neurons and sciatic nerves were used for studying axonal transport. ${ }^{18}$ Three-centimeter sections of the sciatic nerve were placed between agarose gel slabs in a chamber in which the nerve ends were submerged in wells containing the collection buffer (Figure 3a). The preparation was then incubated for up to $12 \mathrm{~h}$. We determined whether MAG-Fc and p75 were

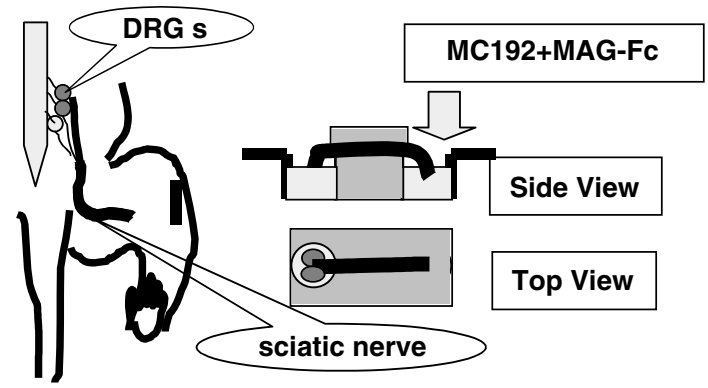

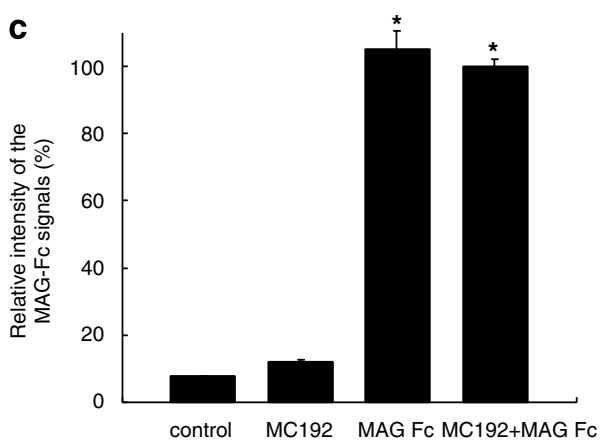

b

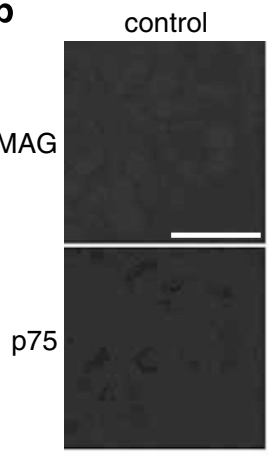

control

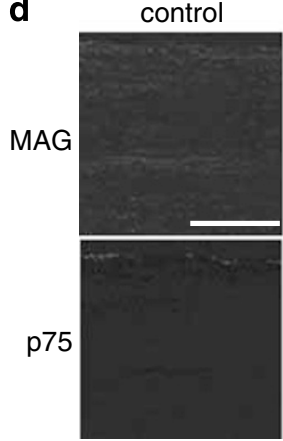

MAG

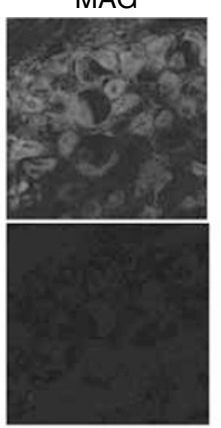

MAG

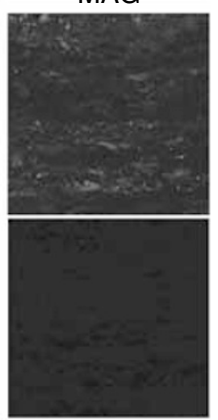

MC192

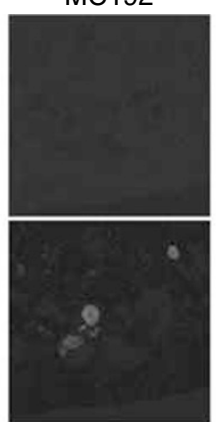

MC192

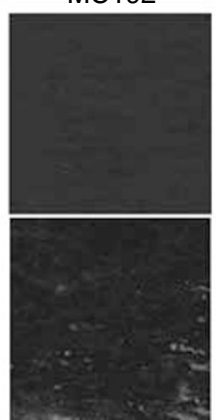

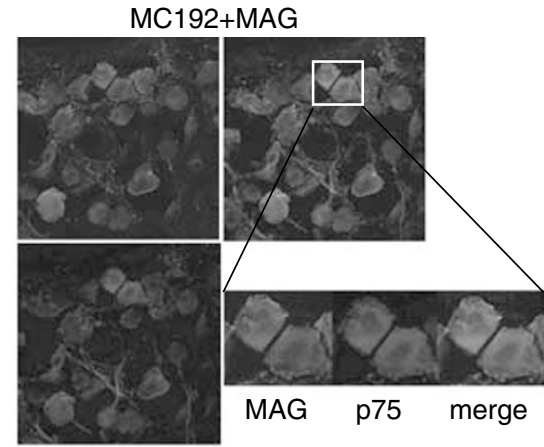

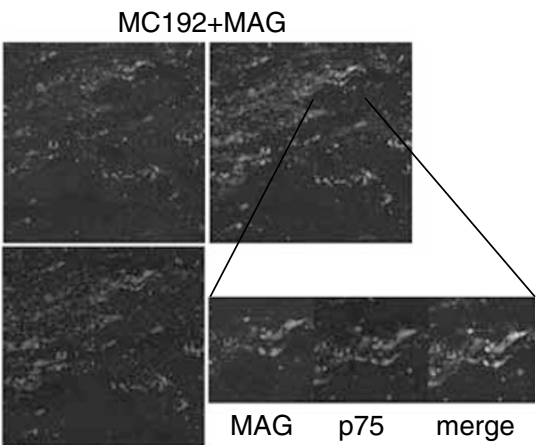

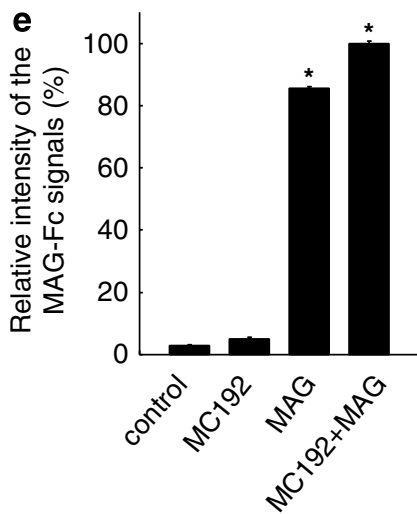

Figure 3 MAG-Fc induces internalization and retrograde transport of $p 75$ and MAG-Fc in the sciatic nerves. (a) DRG-sciatic nerve chamber. The DRGs and sciatic nerves were used for studying axonal transport of p75 and MAG-Fc. The DRG and a 3-cm section of the sciatic nerve were placed between agarose gel slabs in a chamber in which the nerve ends were submerged in wells containing DMEM with $10 \% \mathrm{FCS}$. The distal ends of the sciatic nerves were pretreated with or without MC192 followed by incubation with MAG-Fc. (b) The DRG neurons were immunostained with anti-mouse IgG to detect the retrogradely transported MC192 (p75) or with the anti-human Fc antibody to detect retrogradely transported MAG-Fc (MAG). The signals for MAG-Fc as well as the internalized p75 were observed in the DRG neurons $12 \mathrm{~h}$ after the MAG-Fc treatment. The upper right figure is a merged image showing the colocalized signals for MAG-Fc and MC192 in the neurons. The lower right figures are high-magnification views. Control, no treatment; MAG, MAG-Fc treatment alone; MC192, labeling with MC192 alone. Scale bar =100 $\mu \mathrm{m}$. (c) Quantification of the relative intensity of the MAG-Fc signals in DRGs compared with the signal intensity for the MAG-Fc plus MC192 treatment. Data are represented as the mean \pm S.E.M. of five independent experiments. ${ }^{*} P<0.01$ compared with the control (two-way analysis of variance (ANOVA) followed by Scheffe's multiple comparison test). (d) The sciatic nerves were immunostained with anti-mouse lgG to detect the retrogradely transported MC192 (p75) and with the anti-human Fc antibody to detect the incorporated MAG-Fc (MAG). MAG-Fc as well as MC192 was present in the axons of the sciatic nerves $3 \mathrm{~h}$ following the application of MAG-Fc at the distal axon tips. Scale bar $=100 \mu \mathrm{m}$. (e) Quantification of the relative intensities of the MAG-Fc signals in sciatic nerves compared with the signal intensity for the MAG-Fc plus MC192 treatment. Data are represented as the mean \pm S.E.M. of five independent experiments. ${ }^{*} P<0.01$ compared with the control (two-way ANOVA followed by Scheffe's multiple comparison test) 
retrogradely transported in the sciatic nerves. We pretreated the distal end of the sciatic nerves with or without MC192 for $30 \mathrm{~min}$ and then washed and treated it with MAG-Fc $(25 \mu \mathrm{g} / \mathrm{ml})$. The localization of the signals for MAG-Fc as well as MC192 in the cell bodies was examined at $12 \mathrm{~h}$ after the application of MAG-Fc. The signal for MAG-Fc was visualized with a fluorescence-conjugated anti-human Fc antibody. There were a number of neuronal cell bodies in the DRG neurons that showed signals for MAG-Fc when treated with MAG-Fc alone or MAG-Fc plus MC192, whereas no MAG-Fc signal above background could be observed in the cell bodies when the distal end of the sciatic nerves was treated with MC192 alone (Figure 3b, c). Colocalization of signals for MAG-Fc and MC192 was observed when the distal end of the sciatic nerves was treated with both, suggesting that p75 is transported with MAG-Fc (Figure 3b). These results suggest that the addition of MAG-Fc at the distal end of the axons elicited prominent transport of p75 and MAG-Fc to the cell bodies. In the midsegment of the sciatic nerves, we detected punctate signals for MAG-Fc $3 \mathrm{~h}$ after the addition of MAG-Fc to the distal end of the axons (Figure 3d). Colocalization of MAG-Fc with MC192 was observed in the axons when treated with MAG-FC and MC192. Again, no MAG-Fc signal could be observed in the neurites when the distal end of the sciatic nerves was treated with MC192 alone (Figure 3d, e). Although majority of the signals colocalized, some of the MAG-Fc signals did not colocalize with the MC192 signals, suggesting again that Troy may be involved in this effect. These results clearly demonstrated that MAG-Fc and p75 were internalized and trafficked to the cell bodies via their axons in the sciatic nerves in vitro.

Next, we performed time-course experiments to measure the p75 transport. The trafficking rate of the fluorescence signals for MC192 in the DRG neurons in the absence of MAG-Fc at the distal end of the sciatic nerves was slow; however, the signal movement was potentiated approximately three times by the addition of MAG-Fc (Figure $4 a, b)$. Thus, although there is a basal level of trafficking of p75 in MC192treated neurons in the control condition, as described previously, ${ }^{15}$ MAG-Fc clearly stimulated the trafficking of p75. Addition of NGF $(50 \mathrm{ng} / \mathrm{ml})$ also stimulated the movement of the MC192 signal (Figure 4a, b). We then employed DRGs (P9) from mice carrying a mutation in the $p 75$ gene to determine whether p75 is required for the transport of MAGFc. As expected, only faint signals for MAG-Fc were observed after the addition of MAG-Fc to DRGs from mice carrying a mutation in p75 (Figure 4c, d).

Rap1 is activated in the DRGs after application of MAGFc to the distal end. Experiments with CGNs suggest that p75 carries activated Rap1 from the periphery to the cell bodies. To test this possibility, we examined whether MAGFc also causes Rap1 activation in the sciatic nerves by an in situ assay. To assess the activity of Rap1 in situ, the GSTfused protein of RalGDS (GST-RalGDS), an effector of Rap1, was used. The activated Rap1 was present in bright puncta distributed along axons in the MAG-Fc-treated nerves (for $3 \mathrm{~h}$ ) (Supplementary Figures $3 \mathrm{Sa}, \mathrm{b}$, and $4 \mathrm{~S}$ ) but not in the Fc-treated or Siglec-9-Fc-treated control nerves (Supplementary Figures 3Sa, b, and 4S). Rap1 was activated in a number of neurons located in the DRGs when the distal axons were treated with MAG-Fc for $12 \mathrm{~h}$, whereas no activation could be seen in the DRG neurons treated with Fc or Siglec-9-Fc (Figure 5a, b, Supplementary Figure 4S). We then employed DRGs from mice carrying a mutation in the $p 75$ gene. Little activation of Rap1 was observed $12 \mathrm{~h}$ after the addition of MAG-Fc to DRGs from mice carrying a mutation in p75 (Figure 5c, d). These results suggest that MAG-Fc, acting at distal axons, signals retrogradely through a mechanism that requires $\mathrm{p} 75$.

Inhibition of Rap1 activation induced by MAG-Fc enhances cell death of the neurons. MAG inhibits neurite outgrowth from the postnatal CGNs, and this effect is mediated by several factors, including RhoA, conventional PKC, and the EGF receptor. ${ }^{6,9}$ These findings prompted us to examine if the effect of MAG-Fc on neurite outgrowth is also dependent on Rap1 activity. We generated TAT-Rap1A DN and TAT-Rap1A DA - DN and DA forms of Rap1A fused with the $\mathrm{N}$-terminal 11-amino-acid protein transduction domain of the human immunodeficiency virus protein TAT $^{19}$ - to enable the protein to gain entry into the cell. Both forms of TAT-Rap1A were efficiently incorporated into all of the treated CGNs (data not shown). Although a consensus has been reached that the DN mutants function in cells by competing with normal Ras family proteins for binding to their guanine nucleotide exchange factors, the $17 \mathrm{~N}$ mutant of Rap1A does not always compete well with normal Rap1 for the guanine nucleotide exchange factors in vitro. ${ }^{20}$ Thus, we determined whether Rap1A DN functioned in the cells we used and found that the addition of TAT-Rap1A DN to the CGNs indeed suppressed the Rap1 activation induced by MAG-Fc (Figure 6a). Transduction of TAT-Rap1A DN did not modulate the effect of MAG-Fc on neurite outgrowth (Supplementary Figure 5S), demonstrating that the activation of Rap1 is not necessary for the inhibitory effect of MAG-Fc on neurite outgrowth. Furthermore, TAT-Rap1A DA did not mimic the effect of MAG-Fc (Supplementary Figure 5S). As shown previously, ${ }^{21}$ addition of MAG-Fc activated extracellular signal-regulated kinases (ERKs) in the CGNs. However, transduction of TAT-Rap1A DN did not modulate the ERKs phosphorylation induced by MAG-Fc (Supplementary Figure 6S), although ERK activation has been implicated in MAG-induced growth inhibition. These results exclude the possibility that Rap1 plays a role in regulating the neurite outgrowth of CGNs.

Interestingly, we noticed that there was a net decrease in the number of cells when the cells were treated with MAG-FC in combination with TAT-Rap1A DN (data not shown). To address the hypothesis that Rap1 is primarily involved in the survival of CGNs when stimulated with MAG, terminal deoxynucleotidyl-transferase-mediated dUTP nick end labeling (TUNEL) staining was performed. The number of TUNEL-positive cells was significantly increased in CGNs that were pretransduced with TAT-Rap1A DN and then treated with MAG-Fc (Figure 6b, c); however, neither the TAT protein nor TAT-Rap1A DN by itself had any proapoptotic effect. These results demonstrate that the activation of Rap1 induced 
by MAG-Fc is required for the cell survival of CGNs when stimulated with MAG-Fc.

Finally, the functional implication of the retrograde signals was assessed using the sciatic nerves. TAT-Rap1A DN was applied to the cell bodies of DRG neurons (Figure $6 \mathrm{~d}$ ). We immunostained DRG neurons with an anti-myc antibody to test for the transduction of the TAT-fused protein and found that TAT-Rap1A DN was efficiently incorporated into DRG neurons (52.8\%; Supplementary Figure $7 \mathrm{~S}$ ). The number of apoptotic cells was assessed by the TUNEL assay. The

a

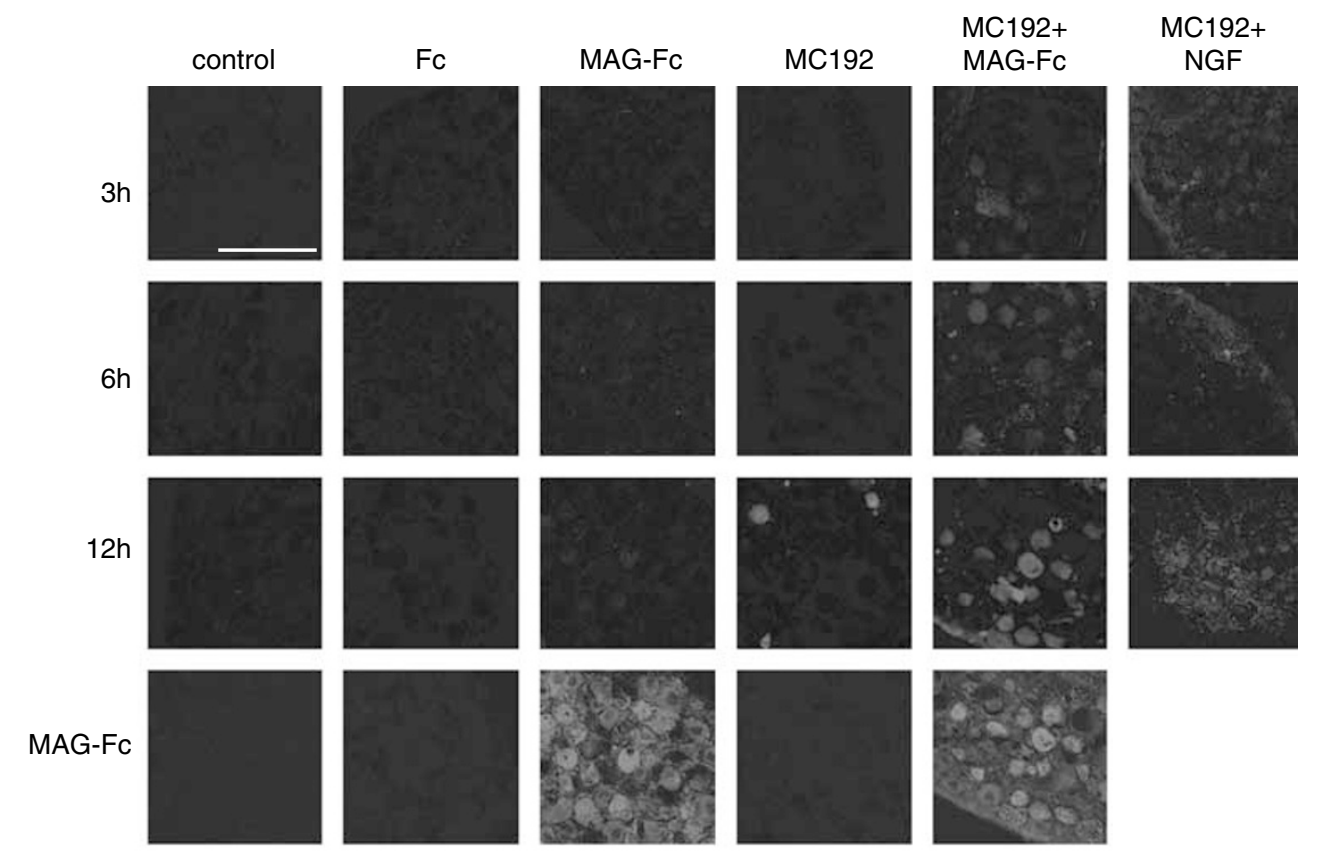

b
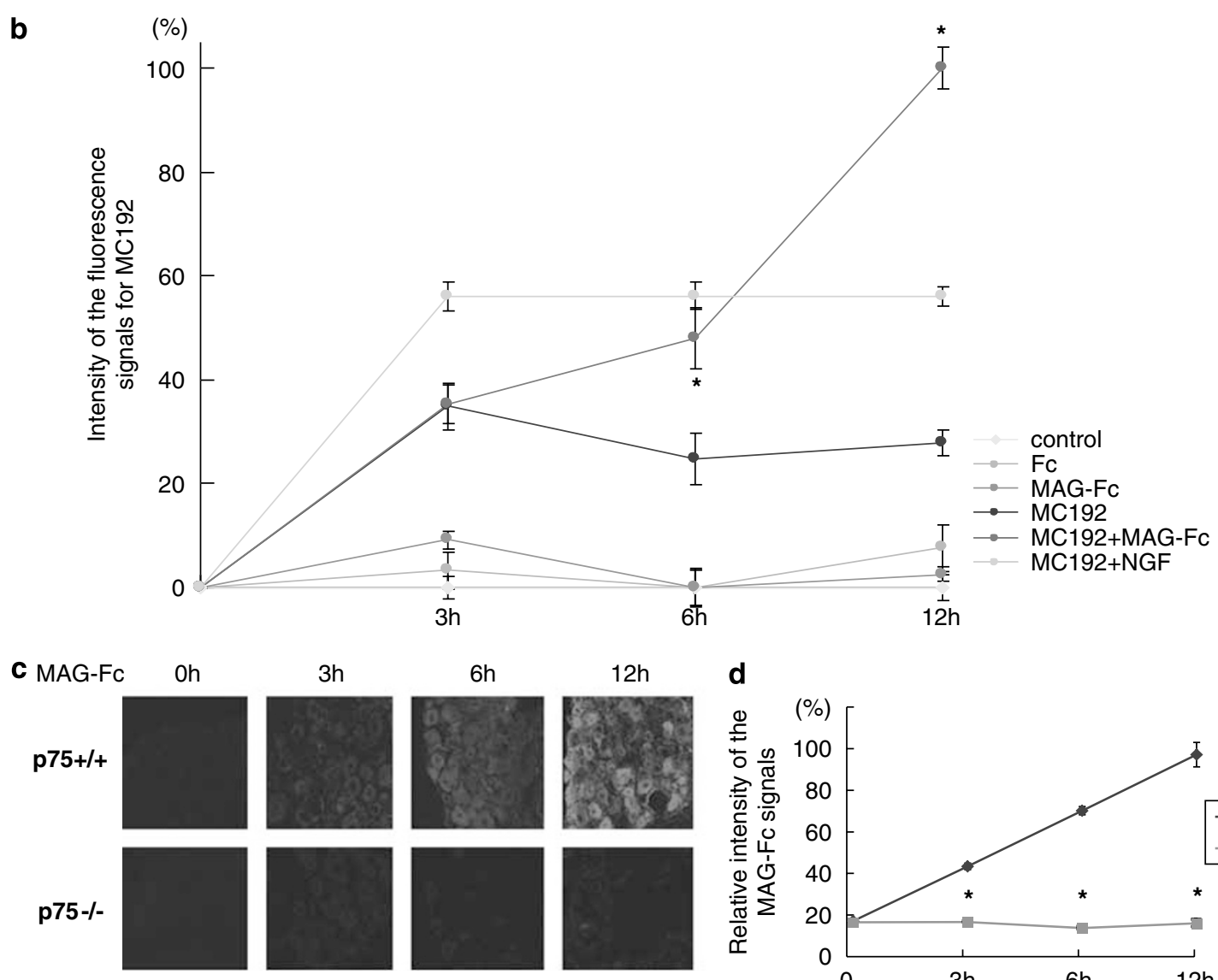

$6 h$
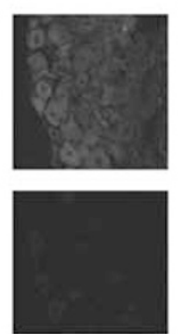

$12 \mathrm{~h}$
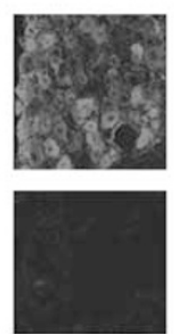

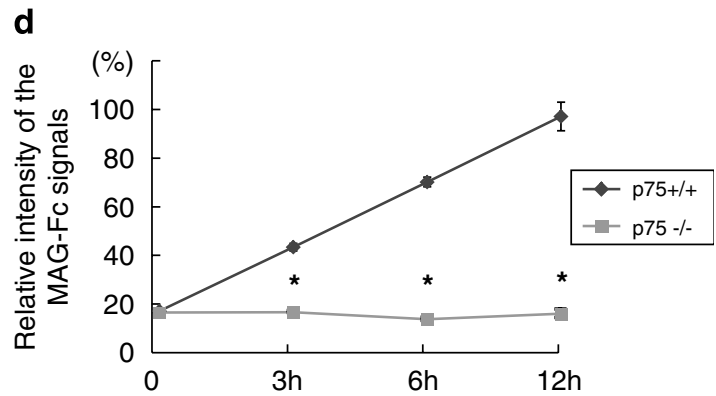


number of TUNEL-positive neurons was slightly increased when treated with TAT-Rap1A DN compared to the controls (Figure 6e). Although MAG-Fc did not have any proapoptotic activity by itself, pretreatment with TAT-Rap1A DN signifi- cantly enhanced the number of MAG-induced TUNELpositive cells (Figure 6e). However, TAT-Rap1A DN did not enhance the number of MAG-induced TUNEL-positive cells in DRGs from mice carrying a mutation in the p75 gene
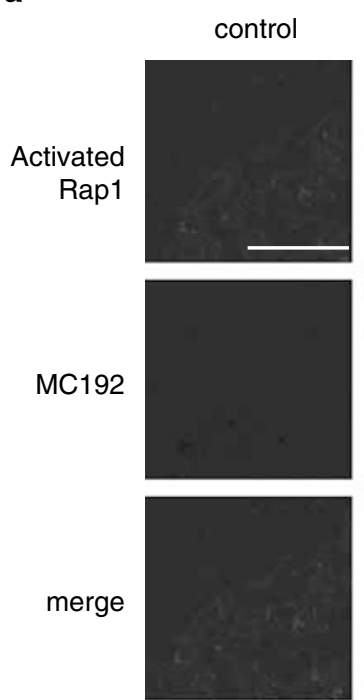

b

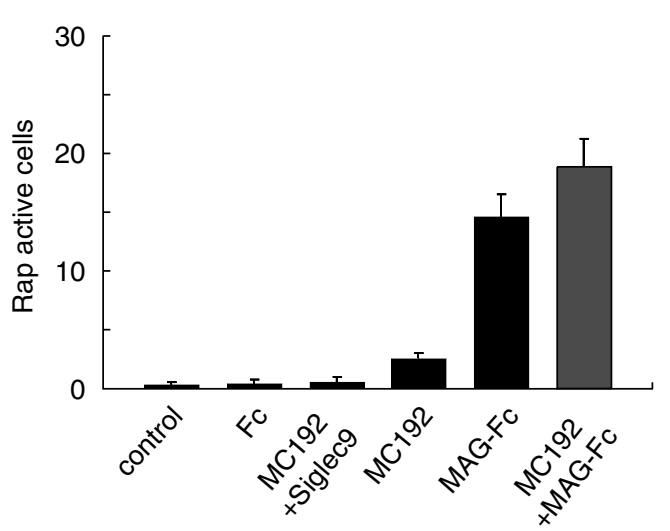

$\mathrm{Fc}$
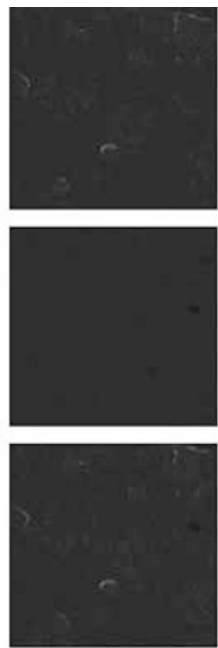

MAG-Fc
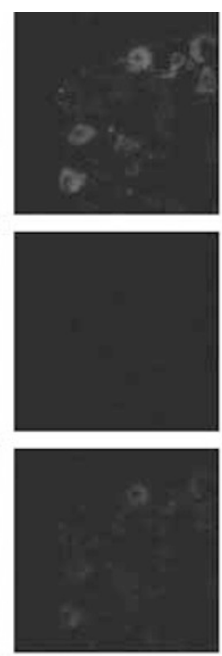

C
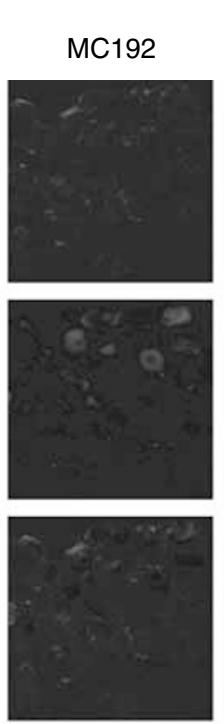

MC192+ Siglec-9-Fc
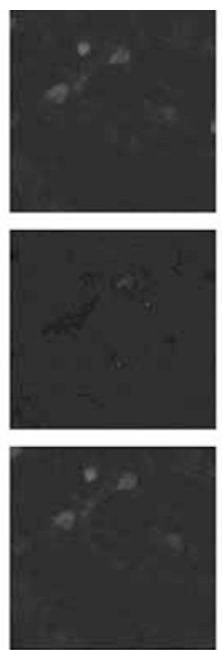

MC192+

MAG-FC
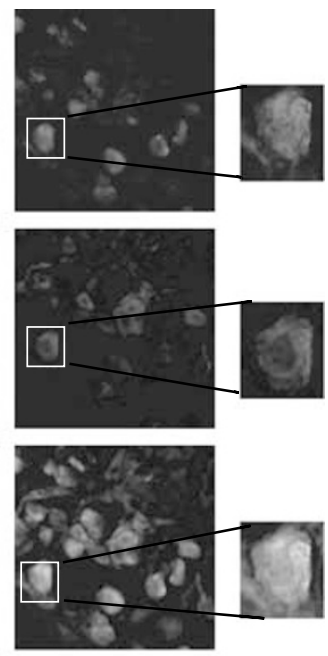

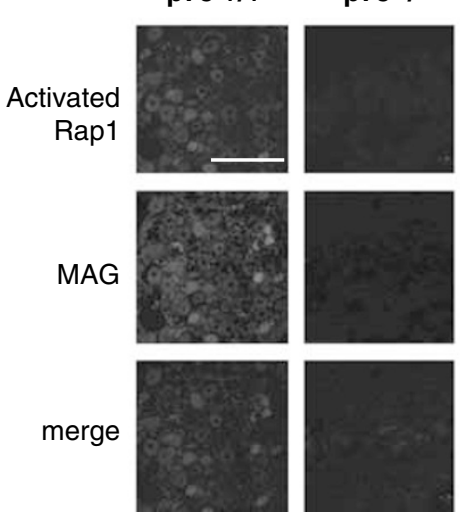

d

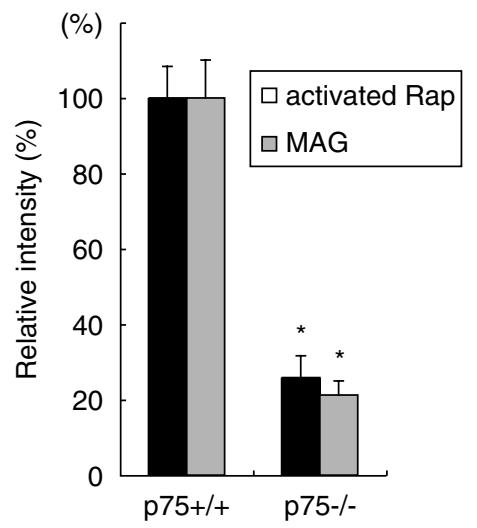

Figure 5 Rap1 is activated in the DRGs after application of MAG-Fc to the distal end of the sciatic nerves. (a) Colocalization of MC192 and activated Rap1 in the cell bodies of the DRG neurons when treated with MAG-Fc for $12 \mathrm{~h}$ at the distal axons. No signals for activated Rap1 (green) were observed in the absence of MAG-Fc or in the presence of Fc or Siglec-9-Fc. MC192, signals for internalized MC192. Scale bar $=100 \mu \mathrm{m}$. (b) The number of the cells with the activated Rap1 in the fixed square of the DRGs. Data are represented as the mean \pm S.E.M. of five independent experiments. ${ }^{*} P<0.01$ compared with the control or MC192 (two-way ANOVA followed by Scheffe's multiple comparison test). (c) Little activation of Rap1 was observed $12 \mathrm{~h}$ after the addition of MAG-Fc in DRGs from mice carrying a mutation in $p 75$ (p75 ${ }^{-1-}$ ). Internalized MAG-Fc (red); activated Rap1 (green). p $75^{+/+}$, wild type. Scale bar $=100 \mu \mathrm{m}$. (d) Relative fluorescence intensities of the activated Rap1 and internalized MAG-Fc in the cell bodies of the DRG neurons. Data are represented as the mean \pm S.E.M. of five independent experiments. ${ }^{*} P<0.01$ compared with wild-type for the activated Rap1 signal or for the MAG-Fc signal (unpaired $t$-test for each factor)

Figure 4 Trafficking of p75 in the DRG sciatic nerves. (a) MC192 was retrogradely transported to the DRG cell bodies from the distal axons when treated with MAG-Fc $(25 \mu \mathrm{g} / \mathrm{ml})$. The distal ends of the sciatic nerves were pretreated with or without MC192 followed by incubation with or without MAG-Fc for the indicated times. The figures represent DRGs immunostained with anti-mouse lgG antibody to detect the internalized MC192 (red). The bottom panels demonstrate the signals for MAG-Fc (green) in the DRG $12 \mathrm{~h}$ after the indicated treatment. Control, no treatment; Fc, Fc treatment alone; MC192 + NGF, labeling with MC192 followed by the addition of NGF (50 ng/ml). Scale $\mathrm{bar}=100 \mu \mathrm{m}$. (b) Relative fluorescence intensities of the MC192 signals in the DRG neurons at the indicated time points. MC192 was transported in the absence of MAG-Fc, but the amount of MC192 transported in the presence of MAG-Fc was approximately three times that transported without MAG-Fc (MC192 alone). Data are represented as the mean \pm S.E.M. of five independent experiments. ${ }^{*} P<0.01$ compared with MC192 alone and control at the corresponding time points (three-way ANOVA followed by Ryan's multiple comparison test). (c) Immunohistochemistry for the detection of MAG-Fc in the DRGs. The retrograde transport of MAG-Fc was observed only faintly in the DRGs from mice carrying a mutation in the $p 75$ gene $\left(p 75^{-1-}\right)$ after the treatment with MAG-Fc $(25 \mu \mathrm{g} / \mathrm{ml})$. p $75^{+1+}$, wild type. Scale bar $=100 \mu \mathrm{m}$. (d) Quantification of the relative intensities of the MAG-Fc signals in the DRG neurons at the indicated time points. Data are represented as the mean \pm S.E.M. of three independent experiments. ${ }^{*} P<0.01$ compared with the wild type at the corresponding time points (two-way ANOVA followed by Scheffe's multiple comparison test) 
a

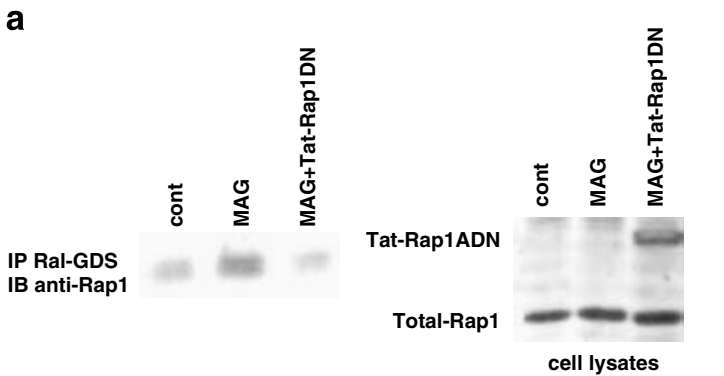

b
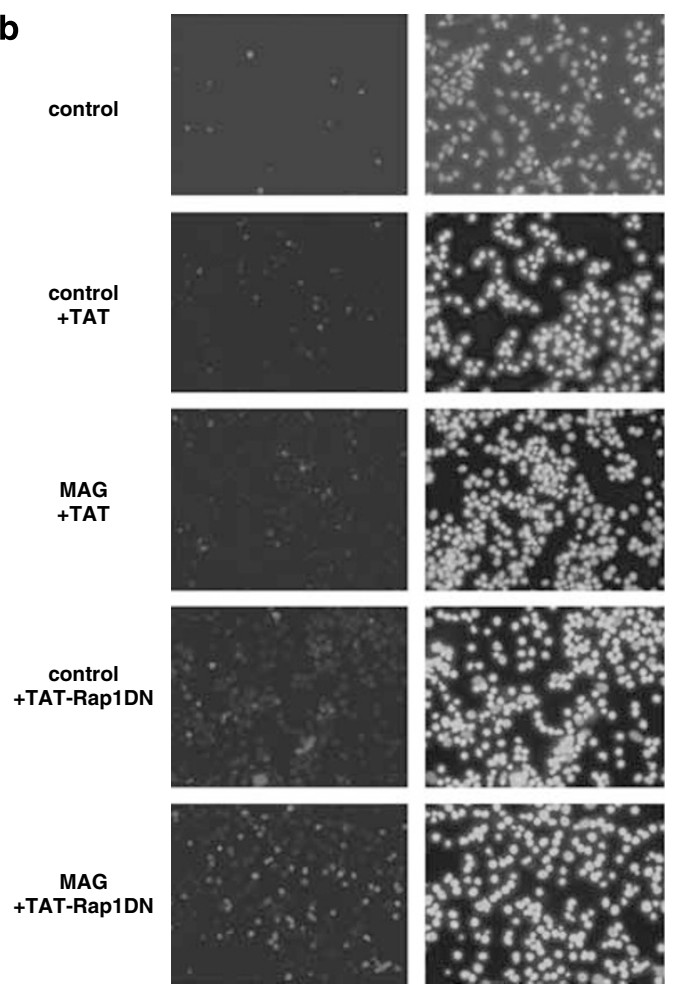

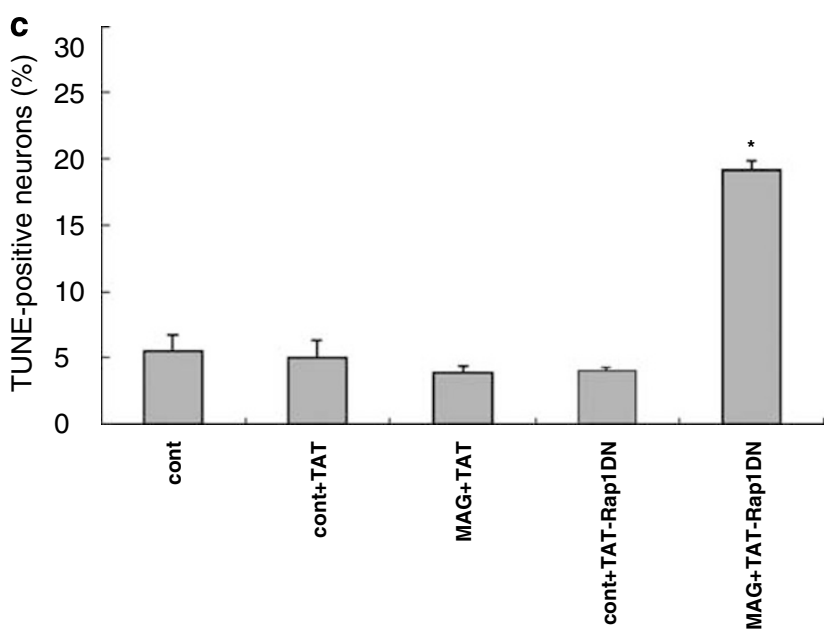

d
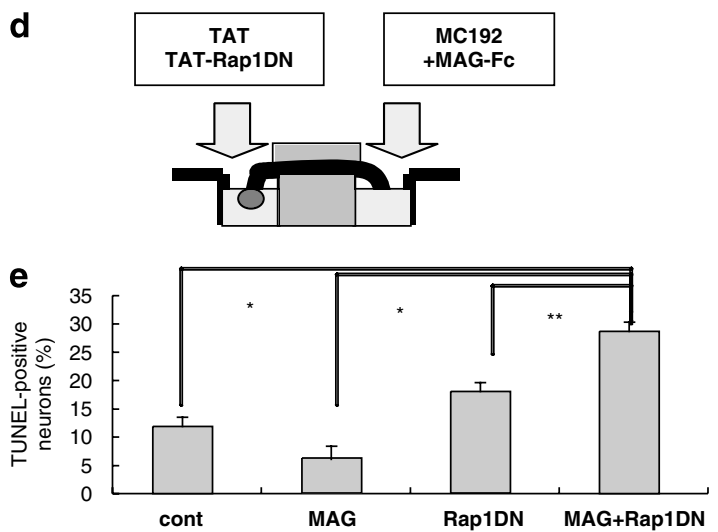

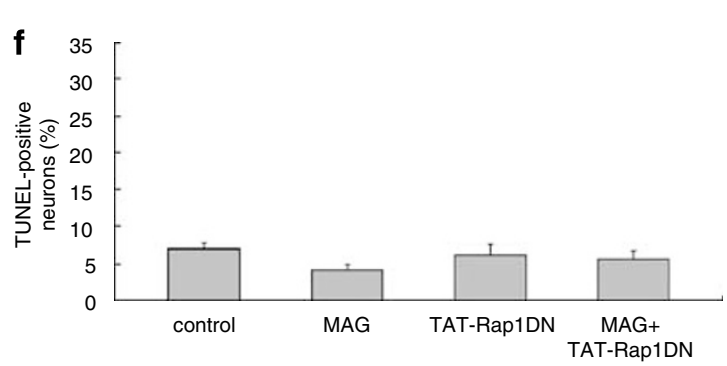

Figure 6 Inactivation of Rap1 elicits MAG-induced cell death of the neurons. (a) Affinity precipitation of Rap1 demonstrates that the Rap1 activation induced by MAG-Fc $(25 \mu \mathrm{g} / \mathrm{ml})$ was attenuated when CGNs were pretreated with TAT-Rap1A DN (left). The right figure shows the total amount of Rap1 and TAT-Rap1A DN in the lysates. (b) TUNEL staining (green) of the CGNs. The CGNs were pretreated with TAT or TAT-Rap1A DN and then treated with or without MAG-Fc. Cell death was assessed $24 \mathrm{~h}$ after MAG-Fc addition. Right panels, nuclear staining with 4,6'-diamidino-2-phenylindole (DAPI). Control, no MAG-Fc treatment; MAG, MAG-Fc treatment. (c) The percentage of TUNEL-positive cells. MAG enhances death of the CGNs when TAT-Rap1A DN is transduced. Results are represented as means \pm S.E.M. $(n=5)$. An asterisk indicates statistical difference $(P<0.01$ compared to control + TAT, MAG + TAT, or control + TATRap1A DN; one-way ANOVA followed by Scheffe's multiple comparison test). Cont, no MAG-Fc treatment; MAG, MAG-Fc treatment. (d) The picture shows the experimental paradigm. TAT-Rap1A DN was applied to the cell bodies of the DRG neurons, and MC192 and MAG-Fc were applied to the distal axons. (e) MAG-Fc induces DRG neuron death when treated with TAT-Rap1A DN. The graph shows the percentage of cells positive for TUNEL. Although MAG-Fc by itself did not have any proapoptotic activity, pretreatment with TAT-Rap1A DN significantly enhanced the number of MAG-induced TUNEL-positive cells. Results are represented as means \pm S.E.M. $(n=5)$. Asterisks indicate statistical significance; ${ }^{*} P<0.001 ;{ }^{* *} P<0.002$; two-way ANOVA followed by Scheffe's multiple comparison test. MAG, MAG-Fc. (f) No significant death of DRG neurons from mice carrying a mutation in the $p 75$ gene was observed when treated with MAG-Fc or TAT-Rap1A DN. The DRGs were pretreated with TAT or TAT-Rap1A DN, and then treated with or without MAG-Fc. Cell death was assessed $24 \mathrm{~h}$ after MAG-Fc addition. The graph shows the percentage of cells positive for TUNEL. Results are represented as means \pm S.E.M. $(n=3)$

(Figure 6f). Thus, MAG-Fc applied to the distal axons of the sciatic nerves induced activation of Rap1 in the axons and the cell bodies of the DRG neurons and inhibition of the Rap1 activity elicited cell death of the DRG neurons when stimulated with MAG-Fc. As TAT-Rap1A DN itself showed slight toxicity in these cells, basal Rap1 activity may be necessary for maintaining cell survival of these neurons. These findings show that activated Rap1, presumably in complex with p75, is trafficked from the distal axons to the cell bodies and that the Rap1 activity induced by MAG-FC is required for the cell survival of DRG neurons and CGNs when stimulated with MAG. 


\section{Discussion}

In the present study, we identified a new signal elicited by MAG. Rap1 is activated in the CGNs and DRG neurons when treated with soluble MAG. In a sciatic nerve explant culture, soluble MAG applied to the distal axons induced internalization and retrograde transport of p75 as well as MAG. p75 appears to be transported with activated Rap1 as the signals for these proteins colocalized and the activated Rap1 associated with p75 in vitro. Rap1 activity was necessary for the cell survival of DRG neurons stimulated with MAG at the distal axons and the MAG-treated CGNs. These findings support a role for p75 and soluble MAG in trafficking the survival signal to the cell bodies.

Internalization and trafficking of p75. Our data demonstrate that MC192 was retrogradely transported in the sciatic nerves. MC192 binds to an extracellular epitope on rat p75 that is distinct from the binding site for NGF. Moreover, MC192 binding does not deleteriously affect NGF signaling in pheochromocytoma (PC12) cells ${ }^{17,22,23}$ or neurons. $^{24}$ Our preliminary data show that MC192 did not influence the effect of MAG on neurite growth (data not shown). It was reported that the internalization of fluorescence-labeled MC192 in the absence of a ligand was slow and equally potentiated by NGF or brain-derived neurotrophic factor. ${ }^{15}$ As these findings were confirmed in our experimental models (Figure 4a, b), MC192 proves to be an excellent tool to analyze the localization of p75 in situ.

Neurotrophin binding to p75 induces internalization through the clathrin-mediated internalization pathway to the recycling endosome. ${ }^{15}$ Ligation of MAG to its receptor complex may regulate interaction with specific docking proteins; these might then sort the receptor to the retrograde transport pathway. Indeed, the association of p75 with the activated Rap1 supports this interesting possibility, and the internalized p75 may carry a signaling complex from the axon terminal to the cell body.

Although MAG typically functions as an intrinsic membrane protein, it was previously shown that soluble MAG, released in abundance from myelin and found in vivo, and MAG-Fc could potently inhibit axonal growth. ${ }^{12,13}$ It is also possible that the transport of p75 in complex with Rap1 does not require the internalization of MAG.

Target-derived signals, which emanate from remote axonal locations, transmit their messages to neuronal cell bodies. Retrograde endosomal signaling in neurons is a multistep process, and the molecular machinery that underlies these events is largely unknown. Retrograde signaling includes the internalization of ligand-receptor complexes in axon terminals, the sorting of complexes into active signaling vesicles, physical translocation of these endosomes along the axonal microtubule network to cell bodies, endosomal signaling, and the dismantling of the retrograde endosomal signaling complex. The molecular mechanisms that govern the retrograde transport of p75 and the endosomal signaling complex should be clarified in the future. Then, we will be able to assess whether retrograde transport of p75 is necessary for its antiapoptotic effect by specific inhibition of p75 transport.
Bidirectional signals elicited by MAG. Our data show that the activation of Rap1 induced by MAG contributes to the cell survival of CGNs as well as DRG neurons. However, MAG by itself had no antiapoptotic activity in the systems used, suggesting that there is an unidentified cell death-inducing signal downstream of MAG. This signal may counteract the Rap1 signal. It was reported that the application of the Rho antagonist C3-05 reduced the number of TUNEL-positive cells by $\sim 50 \%$ in both rats and mice with spinal cord injuries, ${ }^{25}$ suggesting that Rho may be the signal responsible for cell death. Our preliminary data demonstrate that the application of Y-27632 - a Rho-kinase inhibitor - reduced the number of TUNEL-positive CGNs that were pretreated with TAT-Rap1 DN and then incubated with MAG-Fc (data not shown). Therefore, we reason that Rho-kinase and Rap1 may elicit opposite signals and that the balance of these signals might be important for determining the fate of neurons particularly in pathological conditions. The pathological relevance of Rap1 activation by the neurite outgrowth inhibitor MAG is unclear. The activation of Rap1 may be required to maintain viable neurons when they are damaged and exposed to the outgrowth inhibitors in myelin, which triggers neurite retraction by activating Rho/Rhokinase. The crosstalk between the survival signal and the proapoptotic signal needs to be elucidated.

Function of Rap1. Rap1 is implicated in a particularly wide range of biological processes. ${ }^{11}$ It regulates a number of intracellular target proteins and antagonizes Ras. Rap1A is ubiquitously expressed in tissues, whereas Rap1B is predominantly expressed in platelets. The activation of Rap1 was not necessary for the inhibitory effect of MAG on neurite outgrowth in vitro (Supplementary Figure 5S). Rap1 is a critical mediator in the control of integrin activation, and the activation of Rap1 correlates with increased adhesion rather than repulsion. ${ }^{11}$ In our experimental paradigm, however, we did not determine neuronal adhesion.

Rap 1 can bind to and activate the related Raf isoform B-Raf, which subsequently activates ERKs in some cells. In neuronal cells and megakaryocytes, Rap1 induces sustained activation of ERKs. In PC12 cells, the sustained activation of ERKs is required for neuronal differentiation induced by NGF. ${ }^{26,27}$ The activation of Rap1 by NGF requires the internalization of TrkA in intracellular vesicles containing Rap1, B-Raf, MEK, and ERK ${ }^{28}$ Possible downstream signals of Rap1 that contribute to cell survival are ERKs. Indeed, ERKs were activated from $10 \mathrm{~min}$ to $4 \mathrm{~h}$ in the CGNs after treatment with MAG-Fc (data not shown). However, as inhibitors of ERKs enhanced cell death by themselves (data not shown), we could not determine whether activation of ERK by MAG-Fc was necessary for the survival of the neurons in our experimental models.

p75 transduces the signals from MAG, Nogo, and oligodendrocyte-myelin glycoprotein. As other myelin-derived inhibitors are also involved in signaling, including RhoA and conventional PKC, it is suggested that Rap1 is activated by other inhibitors. In addition, another signal transducer of MAG - Troy - may substitute p75. Thus, the functional relevance of MAG-Rap1 in vivo could be assessed by double knockout of p75 and Troy. 
Our observations support the notion that internalized MAG and p75 can act as signaling platforms in neuronal cells and may be incorporated into signaling endosomes to mediate signals that promote neuronal survival.

\section{Materials and Methods}

All experimental procedures were approved by the institutional committee of Chiba University.

Animals. A mouse strain bearing a targeted disruption of the third exon of the p75 gene ${ }^{29}$ in a C57BL/6J background was used; the strain was originally obtained from Jackson Laboratory (Bar Harbor, Maine).

Cell culture. Dorsal root ganglia were removed from P7 rats or P7 mice and dissociated into single cells by incubation with $0.25 \%$ trypsin (Gibco BRL) for $30 \mathrm{~min}$ at $37^{\circ} \mathrm{C}$. For CGNs, cerebella were removed from $\mathrm{P} 7$ rats or P7 mice, mixed with $0.25 \%$ trypsin, triturated, and incubated with $200 \mathrm{U} / \mathrm{ml} \mathrm{DNase} 1$ (TaKaRa) for $15 \mathrm{~min}$ at $37^{\circ} \mathrm{C}$. Dulbecco's modified Eagle's medium (DMEM) (Invitrogen) containing $10 \%$ fetal calf serum was added, and the cells were centrifuged at 1000 r.p.m. for 5 min. Neurons were plated on poly-L-lysine-coated chamber slides. Where indicated, recombinant rat MAG-Fc chimera ( $25 \mu \mathrm{g} / \mathrm{ml}$, R\&D Systems) was added to the medium after plating.

Ras and Rap1 activity assays. Ras or Rap1 activity was assessed by employing the corresponding activation assay kits (Upstate Biotech). Briefly, treated cells were lysed in $\mathrm{Mg}^{2+}$ lysis buffer (MLB buffer) containing $25 \mathrm{mM}$ HEPES, $\mathrm{pH}$ 7.5; $150 \mathrm{mM} \mathrm{NaCl} ; 1 \%$ Igepal CA-630; $10 \mathrm{mM} \mathrm{MgCl}_{2} ; 1 \mathrm{mM}$ EDTA; $2 \%$ glycerol; $2 \mathrm{mM}$ sodium orthovanadate; $1 \mathrm{mM}$ sodium fluoride; and protease inhibitor mixture (Roche Diagnostics). To precipitate active Ras, cell lysates were incubated with $20 \mu \mathrm{l}$ of the Raf-1-Ras-binding domain-agarose conjugate for $30 \mathrm{~min}$ at $4^{\circ} \mathrm{C}$. For Rap1, the Ral-binding domain of the RalGDS-agarose conjugate was used. The beads were washed with MLB buffer and resuspended in $2 \times$ sodium dodecyl sulfate (SDS) sample buffer. Anti-Ras antibody (Upstate Biotech) or anti-Rap1 antibody (BD Bioscience) was used for the blotting.

To assess the activity of Rap1 in situ, GST-RalGDS was used. The tissues were fixed in $4 \%$ paraformaldehyde (PFA) in $0.1 \mathrm{M}$ phosphate buffer (PB) at $4{ }^{\circ} \mathrm{C}$ overnight, rinsed in PB (pH 7.4), and cryoprotected in $30 \%$ sucrose. Cryostat sections $(20 \mu \mathrm{m})$ were incubated with blocking solution containing $5 \%$ bovine serum albumin (BSA) and $0.3 \%$ Triton X-100 in PBS for $1 \mathrm{~h}$, followed by overnight incubation with GST-RalGDS $(5-10 \mu \mathrm{g} / \mathrm{ml})$ at $4^{\circ} \mathrm{C}$. After rinsing, the sections were fixed again in $2 \%$ PFA in $0.1 \mathrm{M} \mathrm{PB}$ for $10 \mathrm{~min}$ at room temperature and were incubated with an anti-GST monoclonal antibody (diluted 1:400 in blocking solution, Santa Cruz Biotech) for $1 \mathrm{~h}$ at room temperature. Alexa 546-conjugated anti-mouse antibody (diluted $1: 400$ in PBS containing $0.1 \%$ Triton X-100, Invitrogen) was used as the secondary antibody. The number of cells with activated Rap1 in a fixed square $(500 \mu \mathrm{m} \times 500 \mu \mathrm{m})$ of the DRG was measured for rat experiments. Five DRGs for each group were measured in an experiment $(n=5)$. Quantitative analysis of fluorescence intensity was performed using the National Institute of Health $(\mathrm{NIH})$ image software, and the relative intensity in a fixed square $(500 \mu \mathrm{m} \times 500 \mu \mathrm{m})$ was measured (the signal intensity at $12 \mathrm{~h}$ after the MC192 plus MAG-Fc treatment in the wild-type mice was estimated as $100 \%$ ). Five DRGs were analyzed for each group in one experiment $(n=5)$.

Coimmunoprecipitation. C-terminal HA-tagged rat $p 75$ and N-terminal myctagged human Rap1 were cloned into a pcDNA3.1 expression plasmid (Invitrogen). $\mathrm{N}$-terminal myc-tagged Rap1A DA (G12V), Rap1A DN (S17N), Rap1B DA (G12V), and Rap1B DN (S17N) were obtained from the UMR cDNA Resource Center, University of Missouri. The plasmids were transfected into 293T cells using Lipofectamine 2000 (Invitrogen), according to the manufacturer's instruction. CGNs from $\mathrm{P} 7$ rats were also used for the detection of endogenous protein interaction. Cells were lysed on ice for $20 \mathrm{~min}$ with lysis buffer $(10 \mathrm{mM}$ Tris- $\mathrm{HCl}(\mathrm{pH} 7.5)$, $150 \mathrm{mM} \mathrm{NaCl}, 1 \mathrm{mM}$ ethylenediaminetetraacetic acid (EDTA), $10 \%$ glycerol, $0.1 \%$ NP-40, $2 \mathrm{mM}$ sodium orthovanadate, $1 \mathrm{mM}$ sodium fluoride, and protease inhibitor mixture). The lysates were centrifuged at $13000 \times g$ for $20 \mathrm{~min}$, and the supernatants were collected. They were then incubated with the anti-HA antibody (for transfected HA-p75; 12CA5, Santa Cruz Biotech.) or anti-p75 antibody (MC192; Chemicon, Temecula, CA, USA) (for CGNs) overnight. Mouse monoclonal IgG
(Sigma) was used as a control antibody. The immunocomplex was collected using Protein G or A Sepharose (Amersham Biosciences). The suspension was centrifuged at $1000 \times g$ for $5 \mathrm{~min}$. The pellets were washed four times with lysis buffer and subjected to SDS-polyacrylamide gel electrophoresis (PAGE), followed by immunoblot analysis by using an anti-myc polyclonal antibody (Santa Cruz Biotech) or an anti-Rap1 rabbit monoclonal antibody (Cell Signaling Tech.). Where indicated, the CGNs were treated with MAG-Fc $(25 \mu \mathrm{g} / \mathrm{ml})$ for 30 or $60 \mathrm{~min}$.

Recombinant protein preparation. Human p75 ICD, HD, or juxtamembrane LR CDNAs were prepared by the polymerase chain reaction, subcloned into the pGEX5X3 vector, ${ }^{14}$ and were expressed in Escherichia colicells BL21. GST and GST-p75 fusion proteins were purified. Briefly, after induction of the proteins with $0.5 \mathrm{mM}$ isopropylthiogalactoside (IPTG) for $3 \mathrm{~h}$, the cells were harvested and sonicated in a lysis buffer containing $50 \mathrm{mM}$ Tris- $\mathrm{HCl}(\mathrm{pH} \mathrm{7.5),1 \%}$ NP40, $1 \mathrm{mM}$ dithiothreitol (DTT), and $1 \mathrm{mM}$ phenylmethylsulfonyl fluoride. After removal of the cell debris, the supernatants were incubated with glutathioneSepharose $4 B$ (GE Healthcare) at $4^{\circ} \mathrm{C}$ for $1 \mathrm{~h}$. After washing with the lysis buffer, GST or GST-p75 fusion proteins bound to glutathione beads were eluted with an elution buffer containing $50 \mathrm{mM}$ Tris- $\mathrm{HCl}(\mathrm{pH} 8.0)$ and $10 \mathrm{mM}$ glutathione in its reduced form.

In vitro binding assay. His-tagged Rap1A protein (cytoskeleton) was loaded with $200 \mathrm{mM}$ GTP $\gamma$ S or GDP in a buffer containing $25 \mathrm{mM}$ Tris- $\mathrm{HCl}(\mathrm{pH} 8.0), 1 \mathrm{mM}$ DTT, $40 \mathrm{mg} / \mathrm{ml} \mathrm{BSA}, 5 \mathrm{mM}$ EDTA, and $0.16 \mathrm{mM} \mathrm{MgCl} 2$ for $20 \mathrm{~min}$ at $30^{\circ} \mathrm{C}$. Then, GTP $\gamma$ S- or GDP-Rap1A or non-loaded Rap1A were incubated with purified GST, purified GST-p75 fusion proteins, or purified GST-RalGDS-Ras-binding domain (RBD) bound to glutathione-Sepharose beads at $4^{\circ} \mathrm{C}$ for $1 \mathrm{~h}$. Beads were washed, and the proteins bound to the resin were subjected to SDS-PAGE, followed by immunoblot analyses.

Internalization assays. The dissociated DRG neurons from P7 rats were cultured overnight in four-well chamber slides. The neurons were incubated for 30 min with Alexa 594-labeled MC192 followed by incubation with Alexa 488labeled MAG-Fc $(25 \mu \mathrm{g} / \mathrm{ml})$ in SFM (DMEM supplemented with B27) at $4^{\circ} \mathrm{C}$. After washing, the neurons were incubated for $60 \mathrm{~min}$ in prewarmed SFM followed by two washes in ice-cold acid $(0.2 \mathrm{M}$ acetic acid and $0.5 \mathrm{M} \mathrm{NaCl})$ to remove the membrane-surface-attached MAG-Fc and were then fixed in 4\% PFA for $30 \mathrm{~min}$. The neurons were examined under confocal microscopy (Olympus FV1000).

The CGNs were incubated on a rocker for $30 \mathrm{~min}$ at $4^{\circ} \mathrm{C}$ in SFM containing MAGFc $(25 \mu \mathrm{g} / \mathrm{ml})$ and MC192. The dishes were washed six times with ice-cold SFM followed by incubation with the prewarmed medium without MAG-Fc and MC192 at $37^{\circ} \mathrm{C}$ for $0,5,15,30$, and $60 \mathrm{~min}$. Endocytosis was stopped by placing the cells on ice, and the cells were washed twice with ice-cold acid ( $0.2 \mathrm{M}$ acetic acid and $0.5 \mathrm{M}$ $\mathrm{NaCl})$ to remove the membrane-surface-attached MAG-FC and MC192. The intracellular fraction was then collected by extracting the cells with lysis buffer. After centrifugation at $15000 \times g$ for $15 \mathrm{~min}$, the supernatant was analyzed using SDSPAGE.

For time-lapse imaging, neurons were plated on the glass bottom of a $35-\mathrm{mm}$ dish and were in situ labeled in SFM with Alexa 594-labeled MC192 $(1 \mu \mathrm{g} / \mathrm{ml})$ at $4{ }^{\circ} \mathrm{C}$ for $30 \mathrm{~min}$. Then, the cells were incubated with prewarmed SFM, including $25 \mu \mathrm{g} / \mathrm{ml}$ MAG-Fc. The fluorescence-labeled p75 was monitored using time-lapse video fluorescence microscopy (Olympus). Five neurons were imaged each time, and the experiments were performed 10 times. We quantified the number of Alexa 594 particles inside the growth cones, neurites, and cell bodies.

DRG-sciatic nerve chamber. We developed a method, based on earlier studies (Delcroix et al., 2003), ${ }^{28}$ in which the DRG neurons and sciatic nerves were used for studying the axonal transport of vesicles. Rats or mice were anesthetized using $\mathrm{CO}_{2}$ inhalation and were decapitated. The L4-5 DRG neurons and the sciatic nerves were harvested. Sections of the sciatic nerves were placed in the collection buffer and transferred to the nerve chamber. Sterile 96-microwell plates (Greiner) were filled with $1 \%$ agarose gel dissolved in sterile PBS. Solidified agar was removed from two wells that were used for collecting transported material. These wells were then filled with $400 \mu \mathrm{l}$ SFM. Sciatic nerve sections were placed on top of the agarose bed, and each end of the section was submerged in a collection well. Most of the exposed portion of the nerve was covered with a thin slice $(1-2 \mathrm{~mm})$ of agarose gel. To prevent dehydration, the plate lid was then placed on top of the chamber in such a way as to avoid contact with the nerve. The chamber was placed in an incubator at $37^{\circ} \mathrm{C}$ for $1-12 \mathrm{~h}$. 
DRG-sciatic nerve axonal transport assay. MC192 $(3 \mu \mathrm{g} / \mathrm{ml})$ was added in the chamber for the distal axons for $30 \mathrm{~min}$ at $37^{\circ} \mathrm{C}$ to label the p75 extracellular domain in the distal axon. The axons were then washed twice and incubated with or without MAG-Fc $(25 \mu \mathrm{g} / \mathrm{ml})$ or Fc $(25 \mu \mathrm{g} / \mathrm{ml})$ in SFM for 3,6 , and $12 \mathrm{~h}$. As an additional control, NGF ( $50 \mathrm{ng} / \mathrm{ml})$ was added instead of MAG-Fc. The tissues were fixed in $4 \% \mathrm{PFA}$ in $0.1 \mathrm{M} \mathrm{PB}(\mathrm{pH} 7.4)$, rinsed, and cryoprotected in $30 \%$ sucrose. Cryostat sections $(20 \mu \mathrm{m})$ were obtained, and MC192 was probed with the Alexa 546-conjugated anti-mouse antibody (diluted 1: 1000 in PBS(-), Invitrogen) or MAG-Fc was probed with the Alexa 488-conjugated anti-human Fc (diluted 1: 1000 in PBS(-), Invitrogen), and examined under confocal microscopy. Quantitative analysis of fluorescence intensity per DRG was performed using the NIH image software, and the relative intensity in a fixed square $(500 \mu \mathrm{m} \times 500 \mu \mathrm{m})$ was measured (the signal intensity at $12 \mathrm{~h}$ after the MC192 plus MAG-Fc treatment was estimated as $100 \%$ ). Ten DRGs for each group were analyzed in one experiment ( $n=5$ or 3). Statistical analysis was done using SigmaStat software. Where indicated, Fc $(25 \mu \mathrm{g} / \mathrm{ml}$; R\&D Systems) or Siglec-9-Fc ( $25 \mu \mathrm{g} / \mathrm{ml}$; R\&D Systems) was added as a control. MC192 treatment was omitted in the experiments using mice.

TUNEL assay. The apoptotic CGNs and DRG neurons were assessed using the ApopTag in situ apoptosis detection kit (Chemicon), according to the manufacturer's instructions. The cells were fixed with 4\% PFA, permeabilized with $0.2 \%$ Triton X-100, washed with PBS, and subjected to the TUNEL assay. The cells were viewed using an Olympus IX71 inverted microscope equipped for epifluorescence. The quantification was performed by counting the TUNEL-positive cells per 200 cells for each group $(n=5)$. Statistical analysis was done using SigmaStat software.

Protein transduction of Rap1. An N-terminal myc-tagged DA human Rap1A (G12V) and DN Rap1A (S17N) were cloned in-frame into the bacterial expression vector pTAT2.1 (a kind gift from Dr. Steven Dowdy) to produce TAT fusion proteins. The vector PTAT2.1 has an N-terminal 6-histidine leader followed by the 11-amino-acid TAT protein transduction domain. Proteins were purified using a nickel-nitrilotriacetic acid (Ni-NTA) column. Briefly, bacterial pellets were resuspended in a buffer containing $100 \mathrm{mM} \mathrm{NaCl}, 20 \mathrm{mM}$ HEPES (pH 8.0), and $8 \mathrm{M}$ urea. They were then sonicated and centrifuged at 12000 r.p.m. for $10 \mathrm{~min}$ at $4{ }^{\circ} \mathrm{C}$. Imidazole was added to the supernatant at a final concentration of $10-20 \mathrm{mM}$. The His-tagged protein was purified in an Ni-NTA column. Bound proteins were eluted with the stepwise addition of 5-10 $\mathrm{ml}$ of imidazole at the concentrations of 50 $100,250,500 \mathrm{mM}$, and $1 \mathrm{M}$. Urea was removed by using desalting PD-10 columns (Sephadex G-25; Amersham Pharmacia Biotech). For transduction of proteins into neurons, TAT-fused proteins were added to the cells at a final concentration of $200 \mathrm{nM}$ in SFM.

Acknowledgements. This work was supported by a Research Grant from the National Institute of Biomedical Innovation (05-12) and Grant-in-Aid for Young Scientists (S) from JSPS. We thank S Dowdy for the PTAT 2.1 vector and BK Mueller for thoughtful comments and critical evaluation of the manuscript.

1. McKerracher L, David S, Jackson DL, Kottis V, Dunn RJ, Braun PE. Identification of myelin-associated glycoprotein as a major myelin-derived inhibitor of neurite growth Neuron 1994; 13: 805-811.

2. Mukhopadhyay G, Doherty P, Walsh FS, Crocker PR, Filbin MT. A novel role for myelinassociated glycoprotein as an inhibitor of axonal regeneration. Neuron 1994; 13: 757-767.

3. Filbin MT. Myelin-associated inhibitors of axonal regeneration in the adult mammalian CNS. Nat Rev Neurosci 2003; 4: 703-713.

4. Yamashita T, Fujitani M, Yamagishi S, Hata K, Mimura F. Multiple signals regulate axon regeneration through the nogo receptor complex. Mol Neurobiol 2005; 32: 105-111.
5. Wang KC, Kim JA, Sivasankaran R, Segal R, He Z. P75 interacts with the Nogo receptor as a co-receptor for Nogo, MAG and OMgp. Nature 2002; 420: 74-78.

6. Yamashita T, Higuchi H, Tohyama M. The p75 receptor transduces the signal from myelinassociated glycoprotein to Rho. J Cell Biol 2002; 157: 565-570.

7. Hasegawa Y, Fujitani M, Hata K, Tohyama M, Yamagishi S, Yamashita T. Promotion of axon regeneration by myelin-associated glycoprotein and Nogo through divergent signals downstream of Gi/G. J Neurosci 2004; 24: 6826-6832.

8. Sivasankaran R, Pei J, Wang KC, Zhang YP, Shields CB, Xu XM et al. PKC mediates inhibitory effects of myelin and chondroitin sulfate proteoglycans on axonal regeneration. Nat Neurosci 2004; 7: 261-268.

9. Koprivica V, Cho KS, Park JB, Yiu G, Atwal J, Gore B et al. EGFR activation mediates inhibition of axon regeneration by myelin and chondroitin sulfate proteoglycans. Science 2005; $310: 106-110$.

10. Yin X, Crawford TO, Griffin JW, Tu P, Lee VM, Li C et al. Myelin-associated glycoprotein is a myelin signal that modulates the caliber of myelinated axons. J Neurosci 1998; 18: 1953-1962.

11. Stork PJ. Does Rap1 deserve a bad Rap? Trends Biochem Sci 2003; 28: 267-275.

12. Tang S, Shen YJ, DeBellard ME, Mukhopadhyay G, Salzer JL, Crocker PR et al. Myelinassociated glycoprotein interacts with neurons via a sialic acid binding site at ARG118 and a distinct neurite inhibition site. J Cell Biol 1997; 138: 1355-1366

13. Tang S, Woodhall RW, Shen YJ, deBellard ME, Saffell JL, Doherty P et al. Soluble myelinassociated glycoprotein (MAG) found in vivo inhibits axonal regeneration. Mol Cell Neurosci 1997; 9: 333-346.

14. Yamashita T, Tohyama M. The p75 receptor acts as a displacement factor that releases Rho from Rho-GDI. Nat Neurosci 2003; 6: 461-467.

15. Bronfman FC, Tcherpakov M, Jovin TM, Fainzilber M. Ligand-induced internalization of the p75 neurotrophin receptor: a slow route to the signaling endosome. J Neurosci 2003; 23: 3209-3220.

16. Kahle $\mathrm{P}$, Hertel $\mathrm{C}$. Nerve growth factor (NGF) receptor on rat glial cell lines. Evidence for NGF internalization via p75NGFR. J Biol Chem 1992; 267: 13917-13923.

17. Chandler CE, Parsons LM, Hosang M, Shooter EM. A monoclonal antibody modulates the interaction of nerve growth factor with PC12 cells. J Biol Chem 1984; 259: 6882-6889.

18. Viancour TA, Kreiter NA. Vesicular fast axonal transport rates in young and old rat axons. Brain Res 1993; 628: 209-217.

19. Schwarze SR, Ho A, Vocero-Akbani A, Dowdy SF. In vivo protein transduction: delivery of a biologically active protein into the mouse. Science 1999; 285: 1569-1572

20. Feig LA. Tools of the trade: use of dominant-inhibitory mutants of Ras-family GTPases. Nat Cell Biol 1999; 1: E25-E27.

21. Dashiell SM, Tanner SL, Pant HC, Quarles RH. Myelin-associated glycoprotein modulates expression and phosphorylation of neuronal cytoskeletal elements and their associated kinases. J Neurochem 2002; 81: 1263-1272.

22. Heckers S, Ohtake T, Wiley RG, Lappi DA, Geula C, Mesulam MM. Complete and selective cholinergic denervation of rat neocortex and hippocampus but not amygdala by an immunotoxin against the p75 NGF receptor. J Neurosci 1994; 14: 1271-1289.

23. Maliartchouk S, Saragovi HU. Optimal nerve growth factor trophic signals mediated by synergy of TrkA and p75 receptor-specific ligands. J Neurosci 1997; 17: 6031-6037.

24. Brann AB, Scott R, Neuberger Y, Abulafia D, Boldin S, Fainzilber M et al. Ceramide signaling downstream of the p75 neurotrophin receptor mediates the effects of nerve growth factor on outgrowth of cultured hippocampal neurons. J Neurosci 1999; 19: 8199-8206.

25. Dubreuil $\mathrm{Cl}$, Winton MJ, McKerracher L. Rho activation patterns after spinal cord injury and the role of activated Rho in apoptosis in the central nervous system. J Cell Biol 2003; 162: 233-243.

26. Wu C, Lai CF, Mobley WC. Nerve growth factor activates persistent Rap1 signaling in endosomes. J Neurosci 2001; 21: 5406-5416.

27. York RD, Molliver DC, Grewal SS, Stenberg PE, McCleskey EW, Stork PJ. Role of phosphoinositide 3-kinase and endocytosis in nerve growth factor-induced extracellular signal-regulated kinase activation via Ras and Rap1. Mol Cell Biol 2000; 20: 8069-8083.

28. Delcroix JD, Valletta JS, Wu C, Hunt SJ, Kowal AS, Mobley WC. NGF signaling in sensory neurons: evidence that early endosomes carry NGF retrograde signals. Neuron 2003; 39: 69-84.

29. Lee KF, Li E, Huber LJ, Landis SC, Sharpe AH, Chao MV et al. Targeted mutation of the gene encoding the low affinity NGF receptor p75 leads to deficits in the peripheral sensory nervous system. Cell 1992; 69: 737-749.

Supplementary Information accompanies the paper on Cell Death and Differentiation website (http://www.nature.com/cdd) 